\title{
Les Écoles en sciences de l'homme : usages indigènes et catégories analytiques*
}

\author{
Olivier Orain $^{* *}$
}

Parmi les mots permettant d'indiquer, voire de mettre en relief, des fonctionnements ou des processus collectifs en histoire des sciences, "école» est sans doute l'un des plus banals. Son évidence et ses ressorts analogiques mettent en perspective la relative rareté des efforts pour l'élaborer, comme s'il n'était pas nécessaire d'en préciser la portée pour l'utiliser ou l'exclure. Sa valeur d'usage et sa transparence supposée font dès lors contraste avec les réserves, voire les polémiques, qu'elle suscite parfois lorsqu'elle devient vulgate pour décrire l'avènement ou la trajectoire d'un groupe circonstancié. Encore faut-il pour ce faire que les réticences qui commentent son usage puissent obtenir une visibilité suffisante : malgré les travaux critiques de Lee Harvey (1987 a et b), Andrew Abbott (1999), Jean-Michel Chapoulie (2001) et Christian Topalov (2003, 2004), l'usage du label « école de Chicago » pour désigner une certaine sociologie américaine demeure extrêmement répandu... Il ne s'agit là que d'un exemple, qui traduit à la fois la prégnance et la durable décote, et même la suspicion de vulgarité, de cet opérateur historiographique. Face aux recours légitimistes et aux réticences qu'il suscite, il nous a semblé utile de le prendre au sérieux, voire au mot, à l'occasion du présent dossier. Ce dernier associe deux articles issus du colloque «Qu'est-ce qu'une école de pensée?» (Dijon, 3-4 novembre 2016), des textes suscités par le comité de rédaction de la Revue d'bistoire des sciences humaines et un entretien avec J.-M. Chapoulie sur la «tradition sociologique de Chicago » à laquelle le sociologue de l'éducation, qu'il est par ailleurs, a toujours refusé d'associer l'étiquette d'école. Avant d'explorer des «cas d'école(s)» grâce aux auteurs des différentes contributions, arrêtons-nous d'abord sur les usages et le pouvoir descriptif de la catégorie.

\section{Usages heureux, usages péjoratifs}

Il n'existe pas d'inventaire et encore moins d'examen systématique des usages de l'expression «école [de] X». On trouve néanmoins quelques tentatives de théorisation ou de montée en généralité. On essaiera d'en rendre compte dans les lignes qui suivent, tout en notant l'impensé que recouvre son usage assez massif (y compris sous la modalité négative: « $[\mathrm{X}]$ ne fut pas une école »), déploré par certains, reconduit le plus souvent. Pour le meilleur ou le pire, le caractère irréfléchi de cet emploi routinisé fait fond sur la dimension quasi-universelle de l'expérience juvénile de l'enseignement (primaire) et de son exercice dans des lieux spécifiques. Que cette universalité soit un mythe et le fruit d'une construction historique devenue pratique très majoritaire importe peu ici ${ }^{1}$. Sur la longue durée du XIX ${ }^{e}$ siècle et des décennies qui lui ont immédiatement succédé s'est néanmoins cristallisé un modèle, avec ses locaux, ses maîtres et ses jeunes élèves - constituant un groupe de pairs —, son dispositif de transmission et de reproduction, etc., dont on peut à bon droit estimer qu'il a constitué un référentiel légitimant pour l'analogie qui nous occupe. Et de même que le sens d' «école» n'a rien d'immuable, ses usages - analogiques ou littéraux - pour parler de collectifs savants seraient à analyser dans leurs traits généraux et leur évolution, autrement dit leur historicité. Cet objectif étant loin d'être atteint $^{2}$, on supposera à titre d'hypothèse leur croissance au XIX ${ }^{e}$ siècle, à hauteur de l'expansion et

\footnotetext{
* Ce texte n'aurait pas vu le jour sans les encouragements et les nombreuses relectures de Wolf Feuerhahn. Mes remerciements vont également à Jacqueline Carroy pour ses précieuses remarques sur un état antérieur de ce texte.

** CNRS, UMR Géographie-cités

${ }^{1}$ Des enquêtes ont montré au demeurant qu'encore au début du XXe siècle, ce sens d' "école » lié à la situation d'enseignement n'était pas encore fixé en France. Cf. l'entretien avec Jean-Michel Chapoulie dans ce dossier.

${ }^{2}$ Un effort de rétrospection reste à faire pour des périodes antérieures au XIX ${ }^{\mathrm{e}}$ siècle, qui montrerait des usages très anciens d'école, notamment pour désigner des groupes de philosophes réunis par une unité doctrinale (dès Diogène

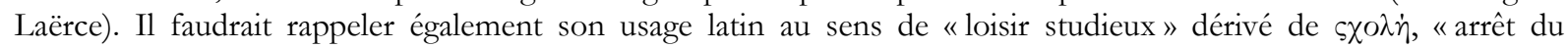


de la différenciation continues du phénomène appelé «science » ou «Wissenschaft» à partir des années 1830 (Carnino, 2015; Hühn, Meier-Oeser, Pulte 2005) et du développement des étiquettes de groupe, propres au monde intellectuel (il en existe plusieurs sortes, cf. infra). Malgré l'«ambiguité » fondamentale que John Servos (1993), l'un des meilleurs connaisseurs de cette histoire, a pu souligner, ces usages sont dans l'ensemble positifs jusqu'au tournant du siècle. J. Servos fait au demeurant longuement référence à l'œuvre monumentale de John Theodore Merz, A History of European Thought in the Nineteenth Century (1902) qui « fait un usage abondant du terme pour décrire les écoles de recherche très spécialisées des laboratoires allemands, les affinités intellectuelles d'individus liés par l'affiliation à une université particulière ou par la citoyenneté d'une ville ou d'une nation, et des traditions de pensée qui traversent les siècles, les frontières

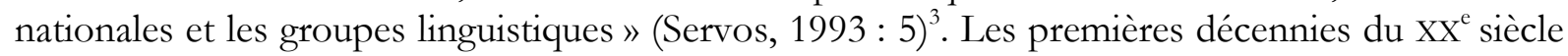
nous apparaissent par contraste comme un moment de bifurcation où des usages nationaux (français tout particulièrement) ou de groupes devenus hégémoniques (dans la «big science » en particulier) en viennent à opposer «la » science, unitaire, consensuelle, et ses périphéries où règnent des pépinières d'écoles livrées à la reproduction médiocre, à l'arbitraire de chefs et à des guerres pichrocolines. J. Servos a relevé quelque chose d'analogue, qu'il analyse comme un legs prégnant « chez les historiens, sociologues et philosophes des sciences du milieu du $\mathrm{XX}^{\mathrm{e}}$ siècle [...] même chez ceux [...] qui accordaient une attention toute particulière à la structure communautaire de la science, tout particulièrement Derek de Solla Price, Diana Crane et Thomas $\mathrm{Kuhn}^{4} »$ (Servos, 1993 : 6). Nous serions pour notre part enclins à mettre l'accent sur la situation hégémonique (même relative) à partir de laquelle ce type d'usage s'observe chez les acteurs de la science, le recours à " école » devenant alors un stigmate. Preuve a contrario : les dominés peuvent le cas échéant retourner ce stigmate dans l'espoir de se rehausser et d'établir une équivalence entre ce qu'ils incarnent et ce que porte le groupe majoritaire ou dominant. De ce point de vue, l'exemple développé par Jacqueline Carroy dans sa contribution à ce volume est éclairant. La promotion d'une " école de Nancy " par Bernheim et ses alliés, contre "l'école de la Salpetrière » ${ }^{5}$ dans les années 1880-90, peut se lire comme la réaction de «provinciaux » contre la construction d'une doxa parisienne dans l'interprétation de l'hypnotisme. Elle répond par ailleurs à ce qui fut aux yeux de Bernheim un usage parisien dont il a retourné le stigmate. On pourrait montrer de manière similaire que la mise en avant du label « école de Grenoble » par Raoul Blanchard et ses élèves dans les années 1930 visait à contester la domination des patrons parisiens de la géographie française, requalifiés en "école de Paris », et s'accompagnait d'un inlassable travail de distinction. Une autre évolution insuffisamment documentée ressortit aux mutations des «années 68 » et au surcroit de doute qu'elles ont jeté sur l'institution scolaire comme modèle, pour un temps synonyme de «bourrage de crâne » et d'aliénation, antinomiques à l'effort - collectif, antihiérarchique - qu'appelait une science alors souvent idéalisée. Le travail opéré par Françoise Waquet dans Les Enfants de Socrate (2008) en prolonge et en systématise, voire en dramatise, l'interprétation : au temps très long, presque atemporel, de la configuration maitre-élève (dont l'école est un variant en même temps qu'une forme d'institutionnalisation) ferait suite une rupture radicale qui signe la fin de cette histoire immobile — c'est le sens de l'épilogue du livre. Au demeurant, F. Waquet la situe moins dans la contestation des années 1970 que dans le développement d'une contractualisation propre au régime plus contemporain des «écoles doctorales ». Ce régime pourrait être interprété comme le dernier état en date d'un processus de

travail ». Il conviendrait également d'examiner les usages du terme en histoire de l'art pour désigner des mouvements artistiques dès le XVII ${ }^{\mathrm{e}}$ siècle (Brückle 2003, 674 ; sur le XIX ${ }^{\mathrm{e}}$ siècle, cf. Le Men, 2016).

${ }_{3}^{3}$ Au demeurant, on trouve aussi chez Merz, note J. Servos, des usages dévalorisants, encore que plus rares. Toutes les traductions de l'anglais proposées sont de nous.

${ }^{4}$ Thomas Kuhn n'utilise « school» dans The Structure of Scientific Revolutions (1962/1970) que pour décrire les situations de « science extraordinaire » pré-paradigmatiques ou « révolutionnaires », mais sans le dédain que J. Servos lui prête.

${ }^{5}$ Voir la contribution de Jacqueline Carroy dans ce volume. 
mise à distance de la «domination rapprochée » d'un «maitre » sur ses « disciples » dont les « années 68 » ont annoncé la crise : lorsque le schème de la paternité « bienveillante » cède la place à un système de prestations engageant des contractants (le «doctorant» et ses encadrants reconnus) dans un système de normes opérant une mise à distance, faire école deviendrait problématique.

Si une certaine désuétude semble frapper les labellisations sur le vif consistant à désigner tel ou tel groupe comme une « école » bic et nunc, il en va différemment en matière d'opérations engageant le passé : le paysage rétrospectif des sciences humaines et sociales est peuplé d'écoles, en nom propre, de personnage ou de lieu, dont l'évocation porte une promesse d'intelligibilité et n'est pas péjorative. À ce titre, «école » est devenue un opérateur historiographique indigène qui interpelle les historiens des sciences par intermittence, les enjoint parfois à en proposer une systématique ou au contraire une réfutation, particulière ou générale. Nous aimerions revenir à ce titre sur trois tentatives d'élaboration selon lesquelles les «écoles » seraient une unité signifiante et significative pour écrire l'histoire des sciences. Leur particularité est de s'être développées de manière autonome, sans références partagées (ou si peu), suivant une logique disciplinaire assez peu soucieuse de ce qui pouvait se produire ailleurs. On les considère comme des lignes de réflexion socialement situées ${ }^{7}$ dans des univers disciplinaires, faites de rebonds et de réactions, ayant par là même une dynamique propre que l'on a essayé de reconstruire sans souci d'exhaustivité.

\section{Clusters ou schools? Quand des sociologues états-uniens se penchent sur l'histoire de leur science à travers des cas d'école(s) (années 1970-80)}

C'est dans le contexte anglo-américain des social sciences, moins hostile a priori à la catégorie de «school» que ne l'étaient leurs homologues « continentaux », que se sont développés des efforts de systématisation, qui semblent à la fois s'inscrire dans un agenda fonctionnaliste et ressortir à une ambiance en quelque sorte post-kuhnienne, tant The Structure of Scientific Revolutions $(1962,1970)$ en constitue une référence cardinale. Certaines tentatives ultérieures ont pris l'habitude d'associer l'ouvrage de Terry Clark ${ }^{a}$ Prophets and patrons, the French University and the Emergence of Social Sciences ${ }^{8}$ (1973) et l'article d'Edward Tiryakian " "The Significance of Schools in the Development of Sociology » (1979) comme une sorte de dyade théorisant la valeur sociologique de la catégorie. Le livre de T. Clark est un tableau historique de la sociologie française vue à travers le prisme de ses groupes d'influence et de la «structure informelle» du système universitaire français, depuis ses "précurseurs» jusqu'aux Durkheimiens. L'article d'E. Tiryakian s'inscrit dans un ouvrage de «métasociologie» (i. e. interrogeant le fonctionnement, les ontologies et les épistémologies disciplinaires) à destination des étudiants avancés et propose une analyse du développement de la sociologie qui fait des « écoles » un opérateur fondamental du fonctionnement disciplinaire.

Cet appariement a ceci d'étrange qu'il correspond moins à une communauté de terminologie ou de préoccupations (sans parler d'intertextualité : Tiryakian ignore royalement le livre de T. Clark dans son article) que de background similaire. Terry Clark réalisa sa thèse sur les sociologies françaises sous la direction de Paul Lazarsfeld (soutenue en 1967) avant de trouver en Edward Shils ${ }^{c}$ et Joseph Ben David des interlocuteurs décisifs pour l'élaboration de son livre de 1973. Edward Tiryakian était déjà un sociologue installé lorsqu'il contribua au livre collectif Contemporary

\footnotetext{
${ }^{6}$ «Mai 68 pourrait bien être d'abord une révolution du privé. Du moins au sens où nous l'entendrons ici : une société dont les membres sont reliés par une relation d'interdépendance puissante, renforcée par le fait qu'ils se trouvent retenus de manière quasi continue, en situation de coprésence physique, dans un lieu relativement unique et clos. Parents/enfants, hommes/femmes, maitres/serviteurs sont liés structurellement au foyer et condamnés au faceà-face. Parce que ces relations y sont en outre structurellement déséquilibrées, on parlera à leur propos de relations de «domination rapprochée ». » (Memmi, $2008: 35$ )

${ }^{7}$ L'enquête s'appuie sur une ébauche de prosopographie dont une partie des résultats a été mise en notes, repoussées en fin de volume afin de ne pas alourdir la lecture.

${ }^{8}$ Il procède pour partie de son travail de PhD (1967) dans le cadre de University of Columbia (outre P. Lazarsfeld sont cités Daniel Bell, Robert Merton et Juan José Linz) mais avec une importante reprise d'enquêtes entre 1967 et 1969.
} 
Issues in Theory and Research. A Metasociological Perspective. Il s'inscrivait partiellement dans la mouvance fonctionnaliste pour avoir fait son $\mathrm{PhD}$ sous la direction de Talcott Parsons, mais à concurrence d'autres affiliations : Pitirim Sorokin, l'existentialisme et la phénoménologie, dont il fut un ardent commentateur et advocate. Nous ferions volontiers l'hypothèse que ces deux tentatives très dissemblables seraient effectivement à replacer dans un cadre fonctionnaliste, encore que significativement tardif. Elles interviennent en effet au moment où ce dernier est ébranlé tout à la fois par la vague contestataire ${ }^{9}$ - américaine puis mondiale — et par le début d'une remise en cause de son hégémonie sur le champ sociologique aux États-Unis. L'exercice de réflexivité historique qu'ils proposent sur les ressorts de la cohésion des communautés de sociologues, marqué par la figure (désormais contestée) du patron charismatique, trouve dans le passé des modèles et des formes de rationalisation : purement sociale, sinon politique, chez $T$. Clark ; plus hybride chez E. Tiryakian, qui mobilise abondamment Kuhn pour donner un plan cognitif à son interprétation.

Ces proximités sont moins frappantes que les différences qui les séparent. La catégorie maîtresse du premier, «cluster» — qu'il avait traduite lui-même par «cercle» dans une publication en français ${ }^{10}$ (Clark, 1971) — se veut moins large qu' "école » pour mieux saisir la performativité politique de groupes d'influence cherchant à trouver une place et à la préserver dans le paysage académique français ${ }^{11}$. De cette éviction découle un travail d'interprétation qui s'intéresse assez peu à l'activité intellectuelle déployée au sein des unités qu'il analyse, de sorte qu'elle pourrait sembler avoir un tout autre objet. Il en va très différemment chez Tiryakian, qui conçoit l'école comme un «type idéal» et comme le moteur d'innovations méthodologiques conçues sur le modèle et dans la démarcation du paradigme kuhnien ${ }^{12}$. Elle s'adosse à une typification fonctionnaliste des « rôles » tenus dans l'école — elle a un «founder-leader » et des «followers » : un «interpreter» de la parole ésotérique du maitre, des «converts» dans une relation de parité générationnelle avec lui, des «lieutenants», des «auxiliaries», des "patrons» (bénéficiaires ou clients). Elle suppose un ancrage «réel», localisé dans le temps et dans des lieux, et un fonctionnement communautaire moins abstrait que celui prêté aux communautés chez Kuhn. En ce sens, située entre l'étude des individus et celle d'ensembles plus vastes, elle lui semble coïncider avec le réquisit mertonien d'une " approach of middle range »" ${ }^{13}$. Elle investit surtout très littéralement l'enjeu professionnel de l'enseignement et de la transmission, et en cela se démarque explicitement du schème de l' "école de pensée » à laquelle elle oppose un usage proche de ceux qui, en histoire de l'art, insistent sur la co-présence et l'interaction rapprochée - entre pairs et avec des élèves au sein d'un atelier. Le parallélisme avec les communautés religieuses (un topos assez banal à l'époque) est quant à lui traité sur un mode relativement neutre, qui abonde une conception plutôt disruptive de l'émergence de nouvelles écoles: elles viennent combler un «vide intellectuel », une situation d'anomie ou de cacophonie, contrastant avec le consensus qui avait prévalu antérieurement. Pour les besoins des trois exemples qu'il développera succinctement dans

\footnotetext{
${ }^{9}$ Des propos récurrents d'E. Tiryakian font état de son saisissement face à la contestation sur les campus américains et son article de 1979 développe (p. 224) des réflexions fugitives sur la "crise vietnamienne aux États-Unis », ses répercussions sur le déroulement du congrès annuel de l'ASA à San Francisco en 1969 et sur «mai 68 » en France, dans lesquels il voit des déclencheurs d'un tournant majeur dans l'histoire de la sociologie. Il a par ailleurs fortement nuancé dans ses nombreux travaux la lecture d'un Durkheim conservateur que l'on trouvait chez Parsons.

${ }^{10}$ Elle correspond largement au chapitre 2 de l'ouvrage dont elle constitue « an earlier version ».

${ }^{11}$ Cette interprétation sera vivement dénoncée par J.-C. Chamboredon : il y voit «la forme neutralisée de la théorie du complot» $(1975,4)$ qui témoigne d'une « vision américaine de la sociologie française » (couverture).

${ }^{12} \mathrm{Il} \mathrm{y}$ aurait beaucoup à dire sur le plan de contenu de sa reprise et les latitudes assez hétérodoxes qu'il s'accorde en la matière. Notons qu'il consacre (sans surprise ?) une place nettement plus extensive que Kuhn aux enjeux de personnalisation / dépersonnalisation dans une « communauté ». Son propos est relativement hybride dans le détail.

${ }^{13}$ Contre la « social theory » très générale et, selon lui, invérifiable de Parsons et ses ambitions totalisantes, Merton a popularisé dans les décennies d'après-guerre la formule "middle range theory », dont la version la plus développée se trouve dans Social Theory and Social Structure (1968). Alors qu'une des intentions de Merton était de bordurer l'ambition explicative des théories sociologiques et leur degré d'abstraction, Tiryakian en fait étrangement une question d'effectif observationnel réaliste pour mener une enquête sur des collectifs.
} 
une dernière partie, E. Tiryakian formule quatre « remarques » qui sont autant de conditions du succès des entreprises qu'il désigne comme des "écoles»: le rôle séminal d'un founder-leader, charismatique certes, mais sensible tout autant à la nécessité de se constituer un vivier d'étudiants et d'alliés; l'adossement à une institution, de préférence centrale (universitaire au sens large, d'excellence et située dans une aire métropolitaine); le rôle stratégique d'une revue comme vecteur de diffusion et de cohésion du collectif; la publication d'un manifeste qui a valeur de «proclamation professionnelle». C'est à l'aune de ces quatre descripteurs qu'il esquisse une présentation de trois écoles implicitement majeures à ses yeux (par opposition aux figures isolées et aux «écoles mineures»): "the Durkheimian School», «the Chicago School» et «the Parsonian School ». Il n'y a pas vraiment de principe d'articulation entre les unes et les autres, mais plutôt un effet de parallélisme qui n'en hiérarchise pas la présentation. À la différence d'autres « parsoniens » plus orthodoxes, et même si l'école de ce dernier est la dernière présentée, son dispositif n'implique aucune espèce de téléologie faisant aboutir la sociologie dans celle-ci.

Cet article d'E. Tiryakian a été abondamment cité et mobilisé par la suite ${ }^{14}$. Il a notamment servi de référence légitimante au travail du sociologue-historien britannique Martin Bulmer ${ }^{\mathrm{d}}$, dont l'ouvrage The Chicago School of Sociology. Institutionalization, Diversity, and the Rise of Sociological Research (1984) a fait date — notamment par son traitement de «the Chicago School» comme une institution et son insistance sur la diversité des approches qui y furent développées ${ }^{15}$. Dans un article intitulé "The Chicago School of Sociology: What Made it a 'School'» (1985), il a par ailleurs éprouvé le besoin de remettre la question de la schoolness sur le métier en la mettant en regard des propositions d'E. Tiryakian. C'est dans une toute autre perspective que son compatriote Lee Harvey s'est référé aux analyses de ce dernier : elles ont constitué pour lui un repoussoir à des titres divers, état d'esprit qui ne s'est jamais exprimé avec autant de vigueur que dans son article "The Nature of 'Schools' in the Sociology of Knowledge: The Case of the 'Chicago School'»(Harvey, 1987a). Dans ce dernier, il met en pièce l'article de Tiryakian en pointant les failles de son usage de Kuhn ${ }^{16}$, le caractère de seconde main de sa documentation et la faible pertinence de ses analyses sur la «soi-disant école de Chicago ». La même année, il a publié une version de sa thèse de doctorat (1985) sous le titre Myths of the Chicago School of Sociology (Harvey, 1987b). Celle-ci est l'occasion d'une autre forme de révision critique, construite autour de « cinq mythes », que l'économie discursive de l'ouvrage entend déboulonner un à un :

Ces cinq mythes sont :

(1) que les sociologues de Chicago étaient initialement des réformateurs sociaux, aux sympathies progressistes ou libérales, et désireux de résoudre des problèmes sociaux. (Chapitre 2)

(2) que la sociologie de Chicago était dogmatiquement qualitative et n'avait aucun intérêt pour les techniques quantitatives de recherche sociale et par suite était ouvertement hostile à leur égard. (Chapitres 3 et 4)

(3) que la sociologie de Chicago n'avait pas d'orientation théorique forte et que sa production a constitué principalement en descriptions. Le genre de théories produit par elle se réduisait à des modèles (typiquement l'organisation urbaine en zones concentriques) à faible pouvoir explicatif. (Chapitre 5)

(4) que la sociologie de Chicago est étroitement associée avec l'interactionnisme symbolique et dominée par la perspective épistémologique de George Herbert Mead. (Chapitre 6)

\footnotetext{
${ }^{14}$ Lee Harvey (1987a) cite une longue série d'émules l'ayant repris de manière a-critique à ses yeux ; Andrew Abbott (1999) s'étonne de la postérité interprétative de Tiryakian sur le cas Chicago, alors que selon lui il a fourni une élaboration générique basée principalement sur une connaissance du durkheimisme et du parsonisme, et n'ayant traité de son troisième exemple que « forcé par la logique de son argumentaire » (Abbott, 1999: 21).

${ }^{15}$ Il a notamment réintroduit dans le paysage des figures «quantitativistes » comme William Ogbun et son élève Samuel Stouffer, mis l'accent sur l'hybridité des moyens d'investigation prônés par les ténors des années 1920-30, etc. ${ }^{16} \mathrm{Il}$ avait publié cinq ans auparavant un article au titre éloquent: "Use and Abuse of Kuhnian Paradigms in the Sociology of Knowledge » (Harvey, 1982), autre exercice de « debunking » (déboulonnage) comme ils sont fréquents dans cette séquence de sa carrière.
} 
(5) que « l'école de Chicago » a dominé la sociologie américaine jusqu'au milieu des années 1930 avant d'entrer en déclin et de s'isoler, principalement en raison d'une approche vieillie et ascientifique de la sociologie. (Chapitre 7) $)^{17}$

La perspective de L. Harvey dans ses travaux des années 1980 n'est pas du tout de rejeter tout usage d'« école» mais au contraire de lui restituer une plus grande dignité conceptuelle, en la replaçant dans une série plus large et articulée d'« unités métascientifiques» — comprenant également «invisible college» et "network» — avec la problématique explicite d'une meilleure saisie des mécanismes de développement et d'évaluation des progrès de la sociologie (et de la science en général). En ceci, Lee Harvey ne dérogeait pas à ce qui avait été une des questions fondamentales de la sociologie des sciences «fonctionnaliste» - depuis Joseph Ben David et Derek De Solla Price au moins. Son insistance sur les processus de croissance met en perspective ses affinités avec l'épistémologie post-poppérienne (Lakatos au premier chef et Kuhn dans une moindre mesure), dont il se départait néanmoins par répugnance pour le caractère ad hoc des reconstructions rationnelles. Il s'est par ailleurs fait le promoteur d'une sociologie historique des sciences examinant au premier chef les processus internes d'évaluation et de discussion " critique » propres à un collectif de recherche, «the development of knowledge through critique » selon ses termes - démarche inspirée de son travail sur la Society for Social Research de Chicago — et qui a comme un parfum poppérien. Si un tel " paradigme » (au sens kuhnien d'exemplar ${ }^{18}$ ) n'a pas fait école, les travaux de L. Harvey, davantage encore que ceux de M. Bulmer, ont en quelque sorte ouvert une séquence révisionniste et plus résolument historiciste. Paradoxalement (ou non ?), ce qu'en a retenu l'historiographie de Chicago $^{19}$ — en faisant complètement l'impasse sur l'heuristique positive d'une « unit approach» ressaisie dans un jeu d'emboîtements institutionnels et de processus dialectiques - est une série de démonstrations en négatif (sur ce que Chicago n'était pas). En cela, le débat sur la schoolness de Chicago apparaît surtout comme un cadre abritant une multiplicité d'enjeux qui ne s'y réduisent pas.

L'une des caractéristiques saillantes de cette historiographie, au-delà de ses différences, est son caractère disciplinaire et comme en miroir : dans une période de crise et d'incertitude marquée par l'effritement du fonctionnalisme, " école » était l'une des modalités envisageable pour réfléchir à l'advenue d'entreprises fécondes de (re)fondation de la sociologie, en un temps anomique ${ }^{20}$. La lecture «tiryakienne» ou "clarkienne» insiste sur le leadership d'un homme s'appuyant sur diverses ressources sociales et intellectuelles, celle des sociologues anglais plutôt sur des opérations collectives égalitaires. Mais «école» n'était finalement qu'un véhicule commode, à concurrence d'autres. Le texte de Monique Hischhorn dans le présent volume inclut une revisite des propositions contenues dans les travaux des deux premiers pour éprouver la «schoolness» d'étiquettes sociologiques reçues comme «école durkheimienne », " école de Le Play », « école de Chicago » et « école de Francfort », non sans un certain scepticisme quant à sa consistance au-delà d'enjeux d'étiquetages. Dans une autre "tradition», propre elle à l'histoire (majoritairement anglophone) des sciences de laboratoire, on voudrait montrer que la consistance se construit sur une lecture beaucoup plus littérale d' " école ».

\section{Le type-idéal de la « research school » en histoire des sciences (1972-1993)}

Dans les années 1990-1992, les historiens des sciences Frederic Holmes ${ }^{\mathrm{f}}$ et Gerald Geison ${ }^{\mathrm{g}}$ ont animé un séminaire de recherche avec un certain nombre de collègues qui attribuaient à la

\footnotetext{
${ }^{17}$ Harvey, 1987b, Introduction.

${ }^{18}$ Dans le Postscript de La Structure des révolutions scientifiques (1970), Kuhn a reformulé sa terminologie en réduisant le sens de "paradigme » à l'idée d’une forme spécifique de résolution des problèmes ("puzzle-solving») et en désignant l'ensemble des caractéristiques structurelles dont cette activité permet la donation comme une «matrice disciplinaire ». L'un de ses éléments essentiels est constitué par les exemples (exemplars) devenus canoniques qui servent de source d'inspiration et de modèle pour de nouvelles activités de recherche.

${ }^{19}$ Cf. notamment Abbott, 1999, chap. 1.

${ }^{20}$ Par contraste, les formes d'interprétation des collectifs sociologiques développées aux USA dans les années 1990 sont beaucoup moins hantées par cette problématique du leadership.
} 
catégorie de «research school» une importante valeur heuristique. Il devait déboucher sur un numéro de la revue Osiris (1993) intitulé «Research Schools: Historical Reappraisals». Ce volume apparaît rétrospectivement comme le climax d'un ensemble de recherches menées par une poignée d'historiens des sciences anglais et états-uniens qui avaient pour dénominateur commun de travailler sur des laboratoires scientifiques (chimie, physique, médecine, physiologie, etc.) fondés et développés au $\mathrm{XIX}^{\mathrm{e}}$ siècle, plus rarement au $\mathrm{XX}^{\mathrm{e}}$, souvent allemands (mais pas exclusivement) et de s'intéresser aux ressorts de leur succès (ou échec) et de leur dynamique collective, notamment sous le prisme de leur capacité à faire école, tout particulièrement à transmettre des savoir-faire pratiques. Le volume publié dans Osiris comprenait sept études de cas encadrées par quatre textes de nature plus réflexive ou «synoptique», dus à John Servos Kathryn Oleskoi, Mary-Jo Nye et Gerald Geison. Il était l'occasion de célébrer un long article de l'historien des sciences anglais Jack Morrell ${ }^{\mathrm{k}}$ paru en 1972 dans la revue Ambix, ${ }^{21}$ "The Chemist Breeders: The Research Schools of Liebig and Thomson » (Morrell, 1972), donné par nombre de contributeurs du volume d'Osiris comme un texte séminal sur la question des "écoles de recherche ». Cet article n'est en l'occurrence pas séparable du travail de commentaire qu'en a donné Gerald Geison dans le livre tiré de sa thèse, Michael Foster and the Cambridge School of Physiology: The Scientific Enterprise in Late Victorian Society (1978), et surtout dans un article épistémologisant de 1981, "Scientific Change, Emerging Specialties, and Research Schools» (Geison, 1981). Il a d'ailleurs proposé dans ce dernier une définition des "écoles de recherche » qui a servi de motto (devise) à cette nébuleuse d'historiens des sciences: "petits groupes de scientifiques chevronnés poursuivant dans un même contexte institutionnel un programme de recherche raisonnablement cohérent aux côtés d'étudiants avancés avec lesquels ils entretiennent des interactions sociales et intellectuelles continues »(Geison, $1981: 23$ ).

À l'occasion de ce volume de 1993, John Servos a proposé une réflexion historiographique très suggestive sur cet objet, la terminologie qui l'entourait et le contexte qui pouvait expliquer le regain d'intérêt pour la catégorie de "school» dans le champ de l'histoire des sciences angloaméricaine des années 1970-80. Après avoir longuement souligné combien le terme était devenu disqualifiant ("derogatory ») pour les hommes de science à la charnière des XIX ${ }^{\mathrm{e}}$ et $\mathrm{XX}^{\mathrm{e}}$ siècles, avant de le devenir également pour les «historiens, sociologues et philosophes des sciences du milieu du Xx ${ }^{\mathrm{e}}$ siècle », il indiquait le retournement amorcé en 1969 avec la publication du compte rendu par Owen Hannaway (1939-2006) du livre de Maurice Crosland, The Society of Arcueil, puis l'efflorescence de l'expression «research school» dans les années 1970, dans le sillage de trois publications - l'article de Jack Morrell, à un bien moindre degré l'ouvrage de Jerome Ravetz', Scientific Knowledge and Its Social Problems (1971) et enfin l'article de Robert Fox, "The Rise and Fall of Laplacian Physics» (1974) — avant de s'interroger sur les raisons de cette «soudaine popularité ». L'argumentaire développé à cet effet possède une richesse qu'il serait fastidieux de résumer mais dont on peut retenir ici certains traits, en ayant à l'esprit combien The Chemist Breeders est construit implicitement comme un exemplar dans la démonstration de J. Servos. Dans un contexte ici aussi post-kuhnien et marqué par la professionnalisation d'historiens de métier en lieu et place de savants ayant développé une érudition historique présentiste — l' "école de recherche » apparait comme une " unité naturelle d'analyse » (Servos, 1993 : 11) particulièrement adaptée à l'étude des recompositions scientifiques du $\mathrm{XIX}^{\mathrm{e}}$ et du premier $\mathrm{XX}^{\mathrm{e}}$ siècles, permettant de développer le comparatisme et de dépasser la vieille antinomie entre histoires «interne» et « externe », mais aussi la perspective consensualiste présente chez T. Kuhn, D. de Solla Price et D. Crane au profit d'une mise en exergue de la conflictualité et des phénomènes de compétition entre collectifs savants. Plus ou moins explicitement, le bénéfice de l'expression consiste à mettre l'accent littéralement sur les processus de formation et d'apprentissage, notamment ceux qui ne sont pas formalisés ou explicités et relèvent d'une transmission de «savoir tacite » des chevronnés aux apprentis - un questionnement surtout mis en avant par Geison en référence aux travaux de

21 Ambix est le journal (publié à partir de 1937) de la Society for the History of Alchemy and Chemistry, fondée en 1935, une société savante créée par des chimistes professionnels. 
Michael Polanyi $(1956,1968)^{22}$. La promotion de la formule «école de recherche» s'accompagnait par ailleurs pour ses utilisateurs d'un affranchissement des «antinomies » entre « originalité » et « reproduction », qui souvent rapprochent le motif de l'école de celui de la secte : « la capacité à être inventif ne peut pas être enseignée comme les tables de multiplication, mais sous un mode adéquat elle peut être cultivée. Dès lors, nombre d'études récentes ont mis l'accent sur l'importance d'un apprentissage rigoureux dans le développement d'un talent créatif exceptionnel. $»^{23}$ (Servos, 1993 : 12). Plusieurs contributeurs du numéro d'Osiris conçoivent cet enjeu de la transmission comme la condition d'un usage de la catégorie en dehors des sciences de laboratoire, dans le cadre d'une histoire de la pédagogie scientifique. C'est d'ailleurs à ce titre qu'elle peut intéresser des historiens des sciences humaines (cf. infra).

L'article de Kathryn M. Olesko, "Tacit Knowledge and School Formation », a une ambition plus spécifique : mettre à plat l'argument cardinal de la transmission de savoir-faire tacites et en opérer une révision critique en en dénonçant le caractère inscrutable, sinon l'ineffabilité. À la déconstruction d'une pétition de principe, qui par sa nature même ne pouvait être illustrée, répond une tentative de sa part pour illustrer par un double exemple ce qui pourrait être explicité des procédures de recherche développées et transmises dans un laboratoire. Et de conclure à la coexistence d'éléments explicites et implicites, leur divulgation et leur publicisation pouvant autant contribuer à la dissolution qu'à la promotion hégémonique d'une « école de recherche ». À un degré plus élevé de généralité, la question du savoir tacite ouvrait sur celle du «style (collectif) de recherche ", central dans l'article de Mary-Jo Nye, "National Styles ? French and English Chemistry in the Nineteenth and Earl Twentieth Centuries ", qui saisit précisément ce moment critique où le style d'une école se dilue et devient par adoption généralisée «style national ». Et de faire l'hypothèse que dans un contexte de nationalisme intense, la suspicion généralisée à l'encontre du caractère scolastique et préscientifique (car polémique) des institutions réunissant maître et élèves pouvait être dépassé en accédant à une dimension d'identité nationale. Cette articulation d'échelles d'observation s'inscrivait parfaitement dans le "programme » formulé par Gerald Geison en 1981 et répondait à des réquisits implicites de l'enquête livrée par Jack Morrell deux décennies auparavant.

Revenir brièvement à «The Chemist Breeders: The Research Schools of Liebig and Thomson» permet à la fois d'apprécier ce qui rend un texte iconique et de mesurer l'écart existant entre ses propositions et les élaborations qui s'en sont ultérieurement revendiquées. Nous avons indiqué sa publication dans une revue érudite d'histoire de la chimie, Ambix; sa forme très particulière de référencialité en découle certainement: elle ne renvoie jamais aux débats contemporains des études sur la science mais exclusivement à l'histoire de la chimie dans sa modalité érudite. L'article divulgue en annexes un certain nombre de documents inédits sur le laboratoire de Justus Liebig à Giessen, parangon des sciences de laboratoire du XIX ${ }^{\mathrm{e}}$ siècle, que l'on imagine précieux aux yeux des amateurs éclairés. Mais surtout, il raconte par longues plages narratives les différentes actions qui ont permis d'instituer les protocoles de recherche, les pratiques pédagogiques et les formes de financement qui ont fait le succès retentissant d'une entreprise savante, alors que par un jeu de parallélismes et de contraste, les efforts contemporains de Thomas Thomson à l'université de Glasgow dessinent une succession d'infortunes et de choix perdants. Le système comparatif très orchestré et stylisé, jugé rétrospectivement "sommaire » (sketchy) par Morrel lui-même (1993), possède néanmoins une efficacité discursive indéniable. Ce va-et-vient entre deux cas contribue à l'hybridité d'un texte qui en un sens procède d'une attente narrative et conjointement entend l'inscrire dans une procédure raisonnée. J. Morrell revendique en effet l'élaboration d'un «modèle » pour interpréter le succès ou l'échec d'une entreprise

\footnotetext{
${ }^{22}$ Autant J. Morrell avait pu réfléchir à la dimension pratique des savoirs dont Liebig (et Thomson à un moindre degré) avai(en)t nourri ses (leurs) disciples, autant la question du « tacit knowledge » n'est pas explicite chez lui.

${ }_{23}$ D'où l'importance des leçons tirées d'une forme de guidance personnalisée qui en quelque sorte prolonge et personnalise l'idée de puzzle-solving dont on sait combien elle est centrale chez Kuhn, autre penseur de la science comme un apprentissage.
} 
savante: c'est tout l'enjeu de la première partie, qui propose un ensemble de schèmes interprétatifs donnés a priori. Ces derniers déterminent le chapitrage de l'étude empirique comparative qui fait suite, en sept angles ménageant une subtile progression temporelle ${ }^{24}$. Ils accordent une importance certaine à la relation maître-élève (quoique souvent diffuse) sans pour autant la constituer en crible. C'est que le locus de la démonstration visée était sans doute ailleurs, dans la mise à jour de l'intrication des «éléments intellectuels, techniques, institutionnels, psychologiques et financiers » (Morrell, 1972 : 45) nécessaire à la compréhension du succès ou de l'échec relatif des entreprises de Liebig et Thomson. L'article de Jack Morrell n'en demeure pas moins une tentative discrète de théorisation ${ }^{25}$ appuyée presque entièrement sur des moyens monographiques. Aussi n'est-il pas surprenant qu'elle ait donné lieu à une tentative de systématisation plus décidée sous la plume de Gerald Geison.

Ce dernier a donc publié en 1981 dans History of Science l'article «Scientific Change, Emerging Specialties, and Research Schools », dont nous ne sommes pas loin de penser qu'il a été le prisme dans lequel l'étude comparative de Jack Morrell publiée neuf ans auparavant est devenue la référence princeps d'une activité de recherche spécifique. Il importe toutefois de noter qu'il développe en parallèle un autre objectif: tracer une frontière très nette entre les pratiques et visées respectives des sociologues et des historiens des sciences, au prix de formules assez souvent stéréotypées ${ }^{26}$. L'article met en regard une forme largement réinterprétée des sept angles morrelliens - devenus pour lui un tableau listant quatorze "facteurs de succès ou d'échec » éprouvés sommairement sur sept $\mathrm{cas}^{27}$ — et un autre tableau tiré du livre des sociologues britannique Michael Mulkay et David Edge, Astronomy transformed: the emergence of radio astronomy in Britain (1976), à la suite de quoi il discute longuement ses propres élaborations, ainsi que celles de J. Morrell ${ }^{28}$ et de Mulkay \& Edge. Cela lui permet de faire émerger un certain nombre d'enjeux un peu plus formalisés qu'ils ne l'étaient dans les travaux antérieurs, concernant le pouvoir de discrétisation de la catégorie « research school» (au regard des «emerging specialties» promues par les sociologues qu'il discute), son hétéronomie relative qui appelle des changements d'échelle, sa place parmi le large éventail de "units of analysis» déployées par les études sur la science postkuhniennes, etc. Il insiste tout particulièrement sur ses avantages, liés à sa focalisation sur de "petits groupes localisés de scientifiques»(Geison, 1981: 32, souligné par l'auteur) à travers lesquels l'étude de l'intrication des facteurs "personnels, sociaux, conceptuels et techniques » (idem) serait facilitée. Tout en récusant l'idée d'une quelconque «formule magique » apte à rendre compte globalement de la structuration de la science "moderne», il érige les "écoles de recherche » comme «la forme organisationnelle concrète prédominante dans la science depuis le milieu du XIX ${ }^{\mathrm{e}}$ siècle » (Geison, 1981 : 37).

À travers cet exemple, doublement situé (par ses objets et par le moment particulier de son déploiement), d'une catégorie valorisée par des historiens des sciences anglophones, nous avons essayé de montrer à quelles conditions les usages d' «école » pouvaient faire l'objet d'un début de formalisation. Nous avons indiqué combien son pouvoir heuristique était indexé sur un usage non métaphorique du terme, mettant en avant des formes socialisées de transmission, en particulier d'une raison pratique. Au lieu d'agréger des noms autour d'une ressemblance

\footnotetext{
${ }^{24}$ On pourrait peut-être les résumer en les formulant dans des termes non-morrelliens : la constitution d'une autorité savante; les modalités du recrutement étudiant; la transmission de savoir-faire techniques; les stratégies de promotion éditoriale d'une école de recherche ; la construction d'un pouvoir académique ; le charisme professoral ; les ressources financières allouées à l'entrepreneur par son institution.

${ }^{25}$ Une tentative qui préfigure aussi à quelques années d'intervalle le schème du «traitement symétrique » dont le programme fort allait faire l'une de ses formules centrales.

${ }^{26}$ Tous les sociologues sont amalgamés, qu'ils soient mertoniens ou «edimburghiens », et abusent du schématisme théorique. L’article de Geison est une défense du corps qui pourrait étonner au regard de son itinéraire.

${ }^{27}$ Où sont réinterprétés les analyses de Morrell (1972), Geison (1978), Servos (1976), Crosland (1967), Fox (1974), Holton (1978) et Hannaway (1976).

${ }^{28} \mathrm{Il}$ récuse notamment la pertinence de l'usage de «modèle » par J. Morrell pour qualifier ce qu'il a entrepris dans son article de 1972.
} 
intellectuelle supposée, comme dans l'usage philosophant d' "école de pensée », l' "école de recherche » est une invitation à l'enquête à partir d'un cas d'espèce. Le motto de Geison (cf. supra) présente une souplesse et un caractère de généralité qui invite surtout à spécifier dans quelles configurations il aurait de la pertinence. À condition de repenser les cadres de la transmission de «savoir-faire», ce genre de travail peut certainement s'extraire du lieu «laboratoire» et s'appliquer à d'autres situations dans lesquelles une passation de " gestes » ou de «style » est ou était organisée.

L'enquête de terrain des sciences humaines pourrait être un topos pour éprouver les modalités d'une transmission de cet ordre : si la pratique individuelle en est fort ancienne, sa codification en livres d'instructions l'est moins (dernier quart du XIX ${ }^{e}$ siècle au Royaume-Uni) et sa construction comme un objectif d'apprentissage universitaire réalisé collectivement et in situ, dans un côtoiement entre "chevronnés » et "apprentis », l'est encore moins. Pour la France, ce n'est guère avant les années 1970 qu'ont été organisées des formations à la recherche de ce type, supposant des interactions en situation ou de bilan a posteriori. Les diverses disciplines géographiques nationales en possédaient une forme différente remontant au début du $\mathrm{Xx}^{\mathrm{e}}$ siècle, plus magistrale, moins interactive, inspirée des traditions naturalistes, à travers le rite de l' «excursion ». Marie-Claire Robic a montré combien ces dernières instauraient par l'exemple une certaine façon de lire les paysages en même temps qu'elles répondaient à un certain nombre d'objectifs sociaux (Robic, 1996, 1997). Dans le cadre de «l'école française de géographie» devenue discipline nationale, la «schoolness» d'une telle procédure est relativement évidente. Nous avons de bonnes raisons de penser qu'il y a là une modalité qui pourrait être redéployée pour analyser comment des styles d'enquête et des formes d'interprétation ont pu faire l'objet d'une transmission, plus ou moins implicite, plus ou moins délibérée.

D'autres lieux génériques (le séminaire en particulier) et d'autres formes de pratique ('interprétation de textes, d'images ou de discours, certaines formes de clinique autorisant la présence de tiers) pourraient être examinés alternativement. Il n'est pas certain que l'on puisse nécessairement les associer à une "école» - plutôt qu'à un individu ou à une discipline. Mais dès lors qu'il y a codification ou passation par l'exemple de " manières de faire » susceptibles de distinguer un collectif et d'être reproductibles, la question de la «schoolness» se pose, sans que « école » soit la seule échelle de pertinence envisageable.

À travers cet exemple de l'histoire des sciences de laboratoire et notre esquisse d'une généralisation possible, nous avons mis l'accent sur l'une des deux grandes modalités qui donnent sens à la catégorie d'école : étudier un collectif (savant) dans ses cohésions (relatives) et son organisation, notamment générationnelle - autrement dit comme une unité à la fois sociale, intellectuelle, pratique, pédagogique, etc. L'autre modalité consiste en revanche à ressaisir ces « écoles» dans un paysage diversifié dont elles expriment la différenciation. Comme il n'existe rien de tel dans les travaux des historiens des sciences précédemment évoqués, nous abordons cette configuration à travers un dernier exemple, nourri par quelques travaux d'historiens de la linguistique.

\section{L'école comme dispositif de différenciation en histoire de la linguistique}

En introduction d'un numéro récent de la revue Histoire Épistémologie Langage (2015) intitulé « «Faire école » en linguistique au Xx ${ }^{\mathrm{e}}$ siècle : l'école de Genève », Christian Puech ${ }^{\mathrm{m}}$ a proposé une réflexion générale sur les usages par les acteurs de la "notion». Il insiste tout particulièrement sur le fait qu'elle «présuppose la pluralité (une école n'est identifiable que par différence) »(Puech, 2015: 6, souligné par nous). Cette réflexion ouvre un dossier consacré à « «l'école de Genève » [qui] n'est certainement pas la plus connue ni celle qui a retenu le plus volontiers l'attention.» (idem, 5). On pourrait reformuler l'enjeu dans un souci de contextualisation en précisant que «l'école de linguistique» dont traite ce dossier fut l'une des diverses composantes d'un paysage académique qui, après la consécration de la figure de Ferdinand de Saussure - porteuse d'une « unification (au moins projetée) des principes généraux 
de description des langues »- voit s'affirmer dans les années 1920-30 un ensemble de groupes dûment labellisés « école de... » (Genève, Prague, Copenhague, New-York, Paris...). Et C. Puech de souligner la coexistence d'attitudes critiques à l'égard de ce que véhicule la catégorie (ainsi chez Louis Hjelmslev [1899-1965] pour qui elle correspond «à une professionnalisation dévoyée, antihumaniste » [id., 10]) et d'autres qui l'utilisent comme un descripteur commode, comme son descendant Knud Togeby (1918-1974), dont C. Puech résume et commente une tentative historiographique datant de 1946 :

\begin{abstract}
À «l'École de Paris » (Meillet, Vendryes, Sommerfelt), l'aspect «sociologique » des faits de langue ; à « l'École de Genève » (Bally, Sechehaye), « les abstractions de la grammaire »; à «l'École de Prague » (Troubetzkoy, Jakobson), la phonologie; à «l'École néo-romantique allemande» (Weisberger et Porzig), l'étude des systèmes de signification. À « l'École de Copenhague » enfin (Brondal, Hjelmslev), rejoignant par une boucle de l'histoire les préoccupations des réalistes et nominalistes du moyen âge, la mise en évidence des universaux des formes linguistiques d'une part, et la prise en compte des systèmes linguistiques comme « essais d'interprétation » d'autre part.

Triple avantage d'une telle représentation: la distribution géographique et historique des écoles se superpose harmonieusement à la répartition des domaines de la discipline, la spécificité de l'objet est maintenue dans sa différenciation même et, enfin, le cadre général (les catégories historiques mises en œuvre) garantit l'intégration maximale de la discipline. [...]

On peut penser qu'une telle représentation réalise, dans les limites d'un exposé "de circonstance », l'intégration optimale des variétés de la discipline. On note également qu'elle n'est possible, dans son discours manifeste, qu'à partir d'une référence absolue (une référence qui n'est elle même référée à aucune autre) : Saussure, lieu d'origine et principe de fermeture de la régression dans le temps et de la dispersion dans l'espace. (Puech, 2015 :11, souligné par l'auteur)
\end{abstract}

De manière significative, l'analyse met à distance une opération «cartographique ${ }^{29}$ dont elle pointe la fonction auto-légitimante, manifestant un doute sur sa pertinence historiographique. Et pourtant, elle ne s'arrête pas à un examen d'usages situés (dans le temps et dans l'espace social de la linguistique), puisqu'elle se redéploie ensuite en interrogeant ce qui fonde le développement des " écoles linguistiques », faisant glisser insensiblement la catégorie d’une perspective sociologique à un questionnement historico-épistémologique, dans lequel «école» devient une catégorie historiographique :

C'est l'enjeu de ce dossier de déterminer plus précisément dans quelle mesure, jusqu'à quel point et de quelle manière une école de linguistique est une école au sens où l'on parle d'école esthétique, caractérisée par un «style », d'école de pensée religieuse, caractérisée par un dogme et une orthodoxie, d'école philosophique, caractérisée par une doctrine et un fondateur. Quelles relations la notion d'école entretient-elle avec celle - tellement galvaudée - de paradigme ? Quels liens le développement des sciences du langage entretient-il avec un lieu, une doctrine, un fondateur, un champ ? (Puech, $2015: 12)$

Cette naturalisation de la «notion» survient après un détour référentiel par les travaux d'une sociologue des sciences, Olga Amsterdamska ${ }^{\mathrm{n}}$ qui "proposait sous le titre Schools of Thought: The Developement of Linguistics from Bopp to Saussure ce qui semble bien être le seul ouvrage d'ensemble sur cette approche de l'histoire de la linguistique sous l'angle des "écoles de pensées ».» (Amsterdamska, 1987). Si l'article de C. Puech se réfère longuement et élogieusement à ce travail, notamment pour insister sur le problème que pose une interprétation purement cognitive des « écoles », il ne revient pas en revanche sur un trait qui les unit, à savoir justement l'insistance sur la valeur différentielle des écoles ${ }^{30}$, thèse qui est au cœur de Schools of Thought...

Dans sa version publiée, ce dernier ouvrage comprend une suite de monographies sur des "écoles de pensée» linguistiques échelonnées dans le temps: il part d’une analyse du « développement cognitif» et de «l'institutionnalisation » de la linguistique en Allemagne au XIX ${ }^{\mathrm{e}}$ siècle, avant d'étudier trois « écoles » s'étant affirmées les unes par rapport aux autres, les «néogrammairiens », «la réaction idéaliste » et «la révolution saussurienne de l'intérieur ». Un long premier chapitre d'introduction précède ces études de cas, qui est l'occasion pour l'auteure de

${ }^{29}$ La métaphore n'est pas de C. Puech.

${ }^{30}$ Cette perspective se retrouve également chez Tiryakian, mais ce dernier n'est pas cité par Amsterdamska. 
préciser sa démarche et son recours à l'expression «school of thought» dans le cadre d' « observations théoriques » globales. L'objet d'étude est implicitement constitué comme un cas d'espèce permettant d'illustrer à quel point continuité et discontinuité, visées cognitives et organisation sociale sont enchevêtrées dans le développement de la "connaissance scientifique ». Contre une vision qu'elle juge réductrice de la «contingence » des «situations cognitives », en un mot contre le «relativisme ${ }^{31}$, son propos revendique la pertinence des "scientific idea systems» (une formule à très forte occurrence) comme môle structurant de l'activité de recherche. Des « raisons heuristiques » l'amènent à séparer l'examen des « développements cognitifs » et celui du «contexte social», dans un mouvement qui part toujours des premiers pour aller vers les seconds. L'entre-deux néanmoins revendiqué n'est pas exempt d'une forme de hiérarchisation implicite qui se retrouve dans l'inclination de l'auteure pour la formule «school of thought». Elle emprunte à Geison l'idée selon laquelle on dispose avec «school» d'une échelle d'analyse particulièrement adaptée pour de telles perspectives et elle reprend son motto, tout en cherchant à lui en substituer un autre. Cela suppose une longue recension de la terminologie désignant des groupes dans les études sur la science, de leur connotation changeante et de leurs avantages et inconvénients. Dans le sillage de Kuhn et Polanyi, elle récuse une vision de la science dans laquelle le «criticisme » serait permanent tout en mettant l'accent sur « le caractère oppositionnel des écoles de pensée » (Amsterdamska, 1987:7) : «[elles] développent des systèmes d'idées qui divergent des systèmes qui prévalaient auparavant dans leur champ de référence » (idem, 9). Arrivée à ce point de son élaboration, elle se démarque de Geison par une définition de son cru : « une école de pensée est un groupe d'universitaires (scholars) ou de scientifiques réunis dans leur départ (divergence) commun, à la fois cognitif et social, à l'encontre d'autres écoles de la même discipline ou spécialité, ou de l'ensemble de cette dernière » (ibid., 11). Elle prend bien soin d'indiquer qu'il y a des degrés variables dans une telle dissidence, et que continuités et discontinuités se composent en proportions variables dans le rapport d'une «école de pensée » à ce qui la précède. La typologie des formes de discontinuité (" philosophiques », «théoriques » et "d'objet et de méthode ») permet de mesurer à quel point la thématisation des "divergences cognitives» est prégnante et première dans cette élaboration. C'est d'ailleurs du caractère imparfait des «scientific idea systems» et de leurs failles logiques que procède la nécessité d'opérer des changements et des révisions qui appellent la création de nouvelles « schools of thought». D'un point de vue social, ces dernières sont hétéronomes dans la science moderne, car elles relèvent de disciplines ou de spécialités plus larges, dont elles sont tributaires jusqu’à un certain point ${ }^{32}$. Cette situation pose la question de la négociation d'une autonomie, qui repose sur le capital social ${ }^{33} \mathrm{de}$ son (ses) promoteur(s), et sur la capacité d'un groupe à tirer avantage d'une position centrale (en termes de moyens) ou périphérique ${ }^{34}$ (en termes de latitude).

Cette présentation, bien que brève, permet néanmoins d'apprécier combien on se situe ici dans un usage d' "école» fort éloigné de celui des historiens des sciences de laboratoire, bien qu'ils soient une référence (parmi d'autres ${ }^{35}$ ) pour elle. La question de la transmission des raisons pratiques auprès des publics d'apprentis est très secondaire: une charge ironique contre la « connaissance tacite » à l'incipit du chapitre introductif laisse entrevoir un agacement à l'encontre de ce thème associé aux «science studies». De manière assez frappante, la réflexion d’Amsterdamska évite presque complètement la question du pluralisme de fait régnant dans les

\footnotetext{
${ }^{31}$ Dès la deuxième phrase du premier chapitre, elle écrit : «Il ne suffit pas d'affirmer que les théories scientifiques et les faits sont des constructions contingentes pour rejeter l'empirisme, démontrer le rôle de la connaissance tacite, ou pour adopter une version plus ou moins radicale de relativisme. » (Amsterdamska, $1987: 1$ )

${ }^{32} \mathrm{La}$ formule qu'elle utilise est «dual legitimation system ». Elle suppose une tension entre la légitimation externe (disciplinaire) et interne (propre à l'école), qui repose sur des interactions de nature diverse, des tribunes, etc.

${ }^{33}$ L'expression n'est pas incongrue au regard d'une référence qu’O. Amsterdamska fait à P. Bourdieu, assez critique, autour du couple succession/subversion.

${ }^{34}$ Elle prend l'exemple de Justus Liebig en s’appuyant sur l'article de Jack Morrell précédemment analysé.

35 L’une des originalités de ce travail, liée à des circonstances biographiques évidentes, est sa mobilisation de philosophes des sciences polonais, qui fournissent quelques lignes analytiques à son propos.
} 
sciences humaines, y compris en linguistique. Il faut dire que son livre construit les «schools of thought» comme des entreprises de démarcation, et ce uniquement sur le mode de la succession : la question de la coexistence dans un champ donné de plusieurs groupes, rivaux ou complémentaires, ne l'intéresse qu'à la marge. En creux, ses élaborations suggèrent une conception unitaire de la Science. Par contraste, C. Puech examine vingt-huit ans plus tard, pour une période ultérieure il est vrai, les ressources symboliques permettant aux linguistes du milieu du $\mathrm{XX}^{\mathrm{e}}$ siècle de concilier une représentation unitaire de leur science avec la prise en compte de sa différenciation en groupes divergents, interprétée en termes de division du travail et de spécialisation.

À travers cette troisième ligne de réflexion historiographique sur la catégorie d' "école ", on a voulu mettre en exergue le potentiel de démarcation / différenciation que mettent à profit certains usages de celle-ci. Nous avons déjà eu l'occasion de souligner combien ce recours distinctif était important pour comprendre l'opposition entre " école de Nancy » et "école de la Salpétrière » que travaille la contribution de J. Carroy dans le présent dossier. La contribution de Marie-Claire Robic en indique d'autres modalités, qui caractérisent le processus de constitution et d'affirmation d'une «école de géographie française» dans les années 1880-1910: jeu d'oppositions en 1889-90 autour du legs d'Émile Levasseur, entre «topographes » fidèles de L. Drapeyron et la « jeune école » réunie autour de P. Vidal de la Blache, dans un contexte de débats sur l'enseignement de la géographie à l'école et sur les modalités d'institutionnalisation de la «matière » dans le supérieur; nouvelle configuration oppositionnelle dans les premières années $\mathrm{du} \mathrm{XX}^{\mathrm{e}}$ siècle mettant aux prises divers protagonistes revendiquant plus ou moins ouvertement leur place sur la scène des sciences sociales, Durkheimiens, Leplaysiens, historiens (C. Seignobos, H. Hauser), et donc représentants de «l'école de géographie française ». Le travail proposé dans ce numéro par Jean-Baptiste Devaux sur «l'école d'économie industrielle », plutôt de type "unit analysis», trouve quant à lui un épilogue "différentialiste », lorsqu'il analyse l'échec de cette école par l'émergence d'une « école toulousaine » rivale et dotée d'un capital symbolique bien supérieur. On trouvera dans le livre de Martine Kaluszynski, La République à l'éprewve du crime : la construction du crime comme objet politique, 1880-1920 (2002), un autre exemple de travail sur la fonction différentielle d'une labellisation en "écoles », autour du cas d'Alexandre Lacassagne (1843-1924). Elle souligne sa rupture partielle et «raisonnable» avec les thèses biologisantes de Lombroso. Elle montre le rayonnement du mandarin lyonnais auprès d'un important public étudiant, ses alliances (avec Tarde notamment), l'usage stratégique de la revue Archives d'anthropologie criminelle et des sciences pénales et l'échec relatif que constitua la progressive récupération du champ de la criminologie par le droit.

Étudier un système de différences est en principe complémentaire d'une lecture privilégiant la spécificité d'une "unité d'analyse », qu'elle soit de l'ordre du style, du fonctionnement collectif, ou qu'elle relève d'une autre modalité de singularisation. Et pourtant, la dialectique du " propre » et du "relatif » n'est pas thématisée en tant que telle dans les travaux qui insistent sur les processus de différenciation et de distinction à l'intérieur d'un champ donné, ils examinent assez rarement et pour lui-même le fonctionnement interne de chaque môle qui le constitue. Il demeure difficile de traiter conjointement, et avec le même degré d'approfondissement, lieux (place) de science et espaces (space) disciplinaires ${ }^{36}$. Symptôme parmi d'autres de la difficulté à ériger les « écoles » en une formule catégorielle bien dessinée ?

\section{L'impossible formule?}

$\mathrm{Au}$ terme de cette brève plongée dans trois corpus et trois « lignages » très largement disjoints, il importe désormais d'en amorcer le bilan et d'apporter quelques compléments d'analyse et de rétrospection. Pour autant, il n'est pas dans notre ambition de proposer une «formule »

\footnotetext{
${ }^{36}$ Cette dichotomie place vs space, lieu traité pour ce qu'il est $v$ système relationnel d'interaction entre des lieux, est une topique essentielle de la géographie de ces 60 dernières années, depuis Ullman, 1954. Certains y voient une opposition structurante là où d'autres y lisent une incitation à rechercher leur articulation.
} 
fonctionnant comme une synthèse de la littérature mobilisée précédemment, tant celle-ci diverge par ses ambitions, ses objets ou ses perspectives. Plus profondément, l'un des termes de l'enquête que nous avons conduite est que "école » est un opérateur catégoriel indéterminé et fuyant, qui appelle souvent un complément (comme dans « école de recherche ») et vaut ce qu'il vaut (parfois très peu, parfois beaucoup plus) dans un travail in situ de labellisation, d'éviction, de substantification ou de démarcation d'un collectif - la liste d'opérations n'est pas close. En outre, la profondeur du travail est inégale. À rebours des usages irréfléchis, les tentatives que nous avons données à lire et à découvrir ne sont pourtant pas à l'abri d'une difficulté : tel un château de cartes, l'effort de théorisation d' "école »menace toujours de s'effondrer au regard des exceptions qu'il souffre, des connotations qu'il ne peut évacuer (comme « secte » ${ }^{37}$, « doctrine », etc.), de son caractère peu commensurable avec d'autres élaborations, et surtout de sa profonde hétéronomie, hormis quelques cas d'espèce.

Le signe le plus sûr de cette hétéronomie nous est donné par la collection spectaculaire de corrélats, loin d'être d'exacts synonymes, que l'on peut substituer ou associer à "école », et qui sont souvent utilisés pour préciser le genre d'usage qu'un interprète veut privilégier : "tradition », « lignage », "collège invisible », " discipline », " paradigme», "programme de recherche », 《 réseau », « cercle »/ " cluster», " solidarity group», « scientific comunity», etc., sans parler de ce qui est moins déterminé et moins chargé théoriquement (" groupe » par exemple). L'incessant travail de démarcation lexicale que l'on trouve dans les tentatives de théorisation (ou a minima d'explicitation) d' " école » manifeste un enjeu de stabilisation essentiel. On pourrait envisager de collationner l'ensemble de ce vocabulaire «groupal» dans un thésaurus et de l'indexer sur des auteurs ou des sensibilités partagées, ou encore de réaliser des graphes de cooccurrence faisant ressortir des configurations associatives ou dissociatives et révélant des usages disciplinaires ou relatifs à une obédience particulière. Une telle enquête, pour instructive qu'elle puisse être, ne ferait pas avancer d'un centimètre la question de la décidabilité terminologique, qui demeure le talon d'Achille de toute entreprise de montée en généralité à visée théorique sur le sujet.

Une tentative de Jean-Louis Fabiani ${ }^{\circ}$ pour réfléchir sur la catégorie — souvent citée depuis nous semble exprimer à sa façon la difficulté que nous essayons de cerner. Invité au colloque du cinquantenaire du Centre de recherches historiques de l'École des hautes études en sciences sociales (1999), "Histoire de la recherche collective en sciences sociales au Xx ${ }^{\mathrm{e}}$ siècle ", il en fit un article publié dans les actes (2005) intitulé «Faire école en Sciences Sociales. Un point de vue sociologique », qui sous un angle tant disciplinaire ${ }^{38}$ que de visée, se distingue nettement du reste des contributions, y compris au sein de la rubrique «Mystifications du collectif » dans laquelle il fut classé. Malgré son titre, cette réflexion est loin de traiter d'« école » de manière exclusive ni même centrale. L'objet central en serait plutôt les "collectifs sociologiques", entendus comme " les rapprochements d'individus au sein de groupes définis par une affiliation théorique, un style de recherche ou un ancrage territorial, objets auxquels il faudrait ajouter les formes de la mise en réseau. Groupes définis par: c'est là bien toute la question. Une bonne partie du travail de définition, qui est largement un travail collectif, a lieu dans un après-coup (...)» (Fabiani, 2005 : 191). Dans la suite de l'article, la question des "noms collectifs", tributaire d'une production « historiographique » rétrospective, fait d'abord l'objet d'une succession d'analyses contrastives : la première porte sur la tension entre «singuliers et collectifs » et souligne le caractère synchrone d'élans contraires recherchant la libération de la créativité individuelle ou l'organisation collective d'une recherche (sur le modèle du laboratoire ou du séminaire) dans les sciences humaines françaises du début du $\mathrm{XX}^{\mathrm{e}}$ siècle ; la deuxième résume ${ }^{39}$ la théorisation des «traditions » proposée par le sociologue Randall Collins ${ }^{\mathrm{p}}$ dont elle retient surtout le type «loyaliste (orienté par des

\footnotetext{
${ }^{37}$ Sur l'usage de « secte » en philosophie et histoire de la philosophie, cf. Albrecht 1995.

38 On pourrait dire, en stylisant outrageusement les choses, qu'il s'agit d'un article de sociologue-philosophe ayant l'histoire de la sociologie (et de la philosophie dans une moindre mesure) pour cadre dans un volume d'enquête sur des pratiques d'historiens.

39 À partir d'un article que J.-L. Fabiani avait traduit pour les besoins d'un numéro de la revue Enquête en 1995.
} 
héros) » ${ }^{40}$ et le type «impersonnel ou anonymes », occasion également d'introduire une catégorie complémentaire que mobilise Collins, les lignages ${ }^{41}$. Le reste du texte est une discussion de l'historiographie du durkheimisme (entre autres du livre de T. Clark) à travers le prisme de sa "dimension collective» et de ses lignages ultérieurs, avec l'esquisse d'un parallèle avec le cas «Chicago ». De manière significative, ce texte dialogique conclut qu' «il n'est pas souhaitable de proposer l'esquisse d'un modèle permettant d'analyser la constitution des collectifs en sciences sociales » (idem : 206), préférant les renvoyer aux «conjonctures particulières » qui les ont vus naitre. Dès lors, chaque cas d'école relève d'une pure singularité historique et tout «collectif» requiert un examen spécifique des modalités ayant présidé à sa constitution, son fonctionnement ou sa mémoire.

Face au désenchantement que peut produire une telle perspective ou plus généralement l'examen historiciste des conditions de production d'un groupe savant socialement reconnu - en ce qu'elle contribue in fine à déconstruire la dimension héroïque ou la cohérence idéalisée ${ }^{42}$ de l'histoire des collectifs en en exhibant les rituels d'institution et les chaines d'opérations rétrospectives - le déni et l'ignorance sont fréquents. Plus rares mais très éloquentes sont les tentatives de réenchantement (Pudal, 2005) ou les formes sophistiquées de reconstruction essentialiste. Ce serait une autre façon de lire les derniers chapitres du livre d'Andrew Abbott ${ }^{\mathrm{q}}$, Department and Discipline (1999), souvent considéré comme une déconstruction ${ }^{43}$. En effet, après avoir mis à jour la fabrication culturelle de l'objet "Chicago» dans les années 1950, puis longuement examiné le fonctionnement éditorial de l'American Journal of Sociology (chap. 2-6), ce dernier reconstruit dans le chapitre 7 (reprise d'un article paru en 1997 dans Social Forces et traduit depuis dans Raisons politiques: Abbott, 2015) une formule à même selon lui de dire l'essence intellectuelle de l'école de Chicago, qui résiderait dans un régime d'explication contextualiste, toujours indexé sur un moment et un lieu (area). La force particulière du dispositif consiste à affirmer in fine que W. Ogburn et S. Stouffer (cf. supra) appréhendaient l'analyse statistique par variables - porteuse d'une rupture potentielle - comme un « raccourci » n'altérant en rien le «paradigme » (formule récurrente dans le livre) chicagoan. De manière significative, le dispositif du retournement argumentatif (concession de la légitimité d'une objection puis reformulation d'une thèse qui la retourne et la dépasse) trouve dans le discours philosophique (chez R. Pudal) ou une thèse cohérentiste d'ordre intellectuel un ressort permettant de réinstaurer l'idée que quelque chose de substantiel cimente un groupe dans le temps.

Tout le problème d'une lecture cohérentiste est de rendre raison des moyens permettant de promouvoir une ligne ou un style collectifs, de son apprentissage et de sa reproduction en somme. Dans une libre réflexion parue dans la revue Qualitative Sociology en 1999, soit la même année que Department and Discipline, le sociologue Howard Becker ${ }^{\mathrm{r}}$ a développé une réflexion qui nous semble de nature à réviser les conditions contemporaines du « faire école ». L'article est

\footnotetext{
40 L'esquisse qu'en fait l'auteur est l'occasion d'un bref rapprochement avec «le modèle d'analyse proposé par Edward Tiryakian » (Fabiani, 2005 : 195). Celui-ci a ici un statut de modalité particulière, alors qu'il se voulait un dispositif général d'intelligibilité du paysage sociologique. J.-L. Fabiani ne le réfère en outre qu'aux cas de Durkheim et Parsons, comme s'il ne disait rien sur Chicago.

${ }^{41}$ La scissiparité et le potentiel d'hybridation (ou de nouage) sont des caractéristiques importantes de l'interprétation de R. Collins (1995) : on pourrait la reformuler en disant que chaque tradition repose sur un nom d'usage (de héros ou de lieu ou de geste) qui peut donner lieu à différentes formes d'appropriation rétrospective caractérisées par leur divergence plus ou moins prononcée.

42 Nous avons ici à l'esprit le genre d'opérations que produit le révisionnisme, quand il remet en cause le label d' «école» (L. Harvey, J.-M. Chapoulie) et s'attelle à diversifier et nuancer des représentations standardisées, voire met l'accent sur la production rétrospective de tradition imaginée (C. Topalov). En revanche, il n'est pas sûr que J.-L. Fabiani les voie d'un bon œil, lui qui regrette en conclusion de son article : «La préoccupation affichée pour l'analyse de la constitution de lignages ou de traditions ne doit pas conduire à considérer les groupements en sciences sociales comme de purs artefacts produits par l'accumulation des souvenirs pieux ou par les lectures intéressées ou prédatrices des héritiers autoproclamés. » Il ne cite pas ces auteurs au demeurant à propos de « Chicago ».

43 On retient souvent de ce livre ses aspects décapants et démystifiants, en oubliant qu'il fut aussi un outil de commémoration pour les 100 ans de la sociologie chicagoane.
} 
partiellement d'inspiration autobiographique et revient sur l'organisation du département de sociologie de Chicago dans les années 1950. Reprenant un distinguo de Samuel Gilmore entre «schools of thoubt» et "schools of activity » ${ }^{44}$, il le mobilise pour établir un contraste entre une analyse (relativement stylisée) de la situation de la sociologie française « jusqu'au milieu des années 1970 » et celle de la sociologie à Chicago. Nous voudrions en tirer deux motifs de réflexion plus générale après avoir traduit le passage in extenso.

[Samuel Gilmore] appelle le premier type «écoles de pensée » et énonce qu'elles sont créées de l'extérieur par des voix critiques qui examinent le champ et décident que certaines personnes partagent certaines idées, que leur travail partage certains traits stylistiques, ce en quoi elles constituent une école. Il appelle le second groupe une «école d'activité ». Ce que les membres d'une telle école ont en commun est de travailler ensemble sur des projets pratiques. Par exemple, ils peuvent organiser une série de concerts ensemble, chacun donnant sa musique à jouer, tout en n'étant pas du tout d'accord sur ce que la musique devrait être. Ainsi, des gens peuvent nous sembler rétrospectivement avoir pensé et agi de la même manière mais pourraient n'avoir jamais fonctionné collectivement (école de pensée). Tandis que d'autre ont fait des choses ensemble alors même que leurs idées n'étaient pas congruentes (école d'activité). Une école au sens classique [...] combinerait les deux traits: ses membres penseraient de la même manière et travailleraient ensemble à la réalisation de leurs idées partagées.

Les «chapelles » de la pensée sociologique française, prédominantes jusqu'au milieu des années 1970, pourraient s'approcher d'un tel modèle complet. Mais c'est sans doute un résultat, je serais tenté de dire un artefact, résultant de ce que l'activité sociologique française était organisée en petits groupes de recherche dirigés par des leaders fameux qui étaient en compétition entre eux pour la domination théorique du champ.

La vie sociologique américaine [...] est organisée en départements, qui sont abrités par des institutions d'enseignement, colleges ou universités, qui exigent que leurs unités enseignent toutes les formes de sociologie requises à cet effet, ce qui embrasse une grande variété de styles de travail. Dès lors, les départements américains sont pour une grande part des «écoles de travail» (schools of activity). Ils peuvent difficilement s'afficher comme des "écoles de pensée », et même des efforts fructueux en la matière ont rarement un résultat durable. [...] À toutes les époques de son développement, Chicago fut une école de travail, une organisation qui essayait de couvrir les principales orientations (possibilities) disponibles dans le champ à un instant $\mathrm{t}$ (même quand l'une d'entre elles était dominante pour un temps), dans la perspective de réunir une équipe qui tienne la route (adequate). Le but n'était pas de présenter un front théorique uni mais de s'assurer un volant d'étudiants et de diplômes, de financer des programmes de recherche, et par là d'entretenir la réputation d'un lieu bon à tous les niveaux. (Becker, 1999 : 8)

Depuis les années 1920-30, une profonde évolution morphologique s'est amorcée, qui nécessite de réévaluer sous quelles conditions il peut être diversement heuristique de parler d' «école » en sciences humaines et sociales. Les premières décennies $\mathrm{du} \mathrm{XX}^{\mathrm{e}}$ siècle ont vu des groupes d'universitaires (au sens large) imposer leur hégémonie (voire parfois leur monopole) sur la production de connaissances concernant les individus et les sociétés humaines et leurs émissions (de toute nature). Cette hégémonie a pu s'inscrire dans des structures compactes et hiérarchisées (comme en France ou en Russie/Union Soviétique) ou alternativement polycentriques (comme en Allemagne ou aux États-Unis), avec pour effet dans le premier cas de consacrer durablement une école dûment labellisée, devenue la matrice nationale d'une nouvelle discipline sous l'effet d'un processus d'institutionnalisation (un trait partagé par nombre de «sciences humaines » et «sciences sociales» en France). Néanmoins, le processus de diversification croissante en "spécialités ", «thèmes » et sensibilités ou l'absence d'un puissant système de centralisation ont contribué à l'émergence d'un pluralisme de fait: c'est souvent d'un élargissement thématique qu'en ces domaines s'affirme, corrélativement ou successivement, une évolution méthodologique, voire épistémologique, qui peut avoir parfois des airs de révolution, et a pu être interprétée comme telle ${ }^{45}$. Les établissements d'enseignement supérieur, ayant à des degrés divers la mission

\footnotetext{
${ }^{44}$ Jean-Michel Chapoulie revient également sur ce distinguo dans l'entretien qu'il nous a consacré à l'occasion de ce dossier.

${ }^{45}$ Révolution relative, car à la différence de ce que suggère la formulation kuhnienne, cela ne débouche que très rarement sur un remplacement de paradigme mais plutôt sur une forme de stratification-superposition.
} 
de transmettre une vision synoptique des disciplines «littéraires ", " humaines » ou « sociales » rendant raison de leur diversité d'approches, ont pris en charge plus ou moins précocement le recrutement d'un personnel permettant de refléter celle-ci. Il en a découlé, à des dates variables selon les pays, la production d'une offre kaléidoscopique rendant très improbable le lent façonnement de spécialistes socialisés dans une «école» et favorisant une idéologie de l'hybridation individuelle. S'il reste un espace pour faire école (d'une quelconque manière), c'est de manière plus ou moins incomplète dans les second et troisième cycles universitaire (graduate / postgraduate, écoles doctorales), à concurrence d'injonctions extérieures (ressortissant par exemple à des vogues ou à des solidarités générationnelles) et dans une relation complexe à la commandite.

En opérant ce genre de montée en généralité, nous avons clairement indiqué un parti-pris en faveur d'une conception assez littérale d' "école » qui accorde une attention instituante (ne fûtelle qu'un cadre) aux processus d'enseignement et de transmission. Les situations d'apprentissage sont plus diverses que ce qu'un cadre trop rigidement «scolaire » pourrait suggérer. En revanche, elles sont très certainement la matrice de nombre de "gestes» et de «styles » dont la reproductibilité est organisable selon des modalités diverses : l'interaction entre pairs ou avec des médiateurs est l'une d'elles, sans être la seule. À bien des égards, la formule de la "school of activity» trouve un écho dans la comparaison de Tiryakian avec l'atelier de l'artiste entouré de ses élèves et dans les formulations des historiens des sciences de laboratoire. Elle pourrait valoir aussi pour les sciences qui organisent un apprentissage collectif du «terrain » incluant une interaction in situ ou rétrospective (de débriefing) dans laquelle les barrières sociales sont plus ou moins mises entre parenthèses, comme nous l'avons esquissé précédemment.

Par contraste, on peut s'interroger sur l'intérêt de proroger le schème vieilli de l' "école de pensée », dont la consistance est l'enjeu d'opérations homogénéisantes. D’un point de vue d'historien des sciences, elle est une formule d'acteurs (délégitimante ou valorisante) dont la naturalisation dans l'historiographie est hautement problématique. Elle a pour corrélat les innombrables « ismes » (Boschetti, 2014) qui fleurissent et se fanent depuis le dix-neuvième siècle dans le monde des arts et dans celui des «idées» et des doctrines. Ces derniers sont aussi des objets historiques mais font souvent l'économie de la dénotation spécifique à "école». La contribution d'Annie Petit sur le(s) positivisme(s) dans ce numéro de la RHSH s'inscrit précisément dans une tension entre l'étude d'une «mouvance» et de son processus de ramification, et l'examen de ses tentatives pour transmettre un corps de doctrines et d'attitudes, explicitement adossées à une réflexion sur l'éducation. Elle relit le "positivisme » dans ses expressions changeantes sur un siècle comme une «étiquette » souple ayant agrégé des volontés de faire «école», "parti » et «église » en proportions variables, à travers une succession de scissions, hérésies, et changements d'échelle. Dans ce cas d'espèce, « école » est tantôt très proche de "secte », tantôt de bien d'autres «ismes", à l'étude desquels il se rattache. Les problèmes suscités par l'historicisation de ces derniers ne sont pas moindres et ils pourraient faire tout autant l'objet d'un dossier de la Revue d'bistoire des sciences humaines.

\section{Bilan et envoi}

Arrivé à ce stade de la réflexion, il convient de nuancer le scepticisme désenchanté que pourrait susciter la lecture de cette introduction quant à l'intérêt de la catégorie d' " école ». Tout d'abord, le fait que ce soit une catégorie d'acteurs mobilisée très fréquemment, souvent dans un intense travail territorial, suffit à lui conférer un intérêt pour une histoire sociale ou une sociologie historique des pratiques et représentations savantes. C'est tout l'intérêt des travaux attentifs aux opérations de labellisation que de montrer de manière fine combien l'impatronisation d'un nom collectif passe par des opérations variées, au demeurant pas toujours parfaitement explicites et délibérées. Elle est aussi le révélateur d'un imaginaire savant, par exemple quand elle sert à opposer une universalité scientifique avec des «chapelles» ou des «sectes» scolastiques supposées maintenir jalousement leur entre-soi. Il y aurait par ailleurs du sens à la réévaluer 
comme étiquette propre à résumer pour des contemporains l'action d'un entrepreneur de science particulièrement industrieux. "École de $\mathrm{X}$ » est un nom propre qui ouvre plus ou moins directement (mais avec des exceptions) sur une conception paradoxalement individualiste des collectifs, en ce qu'ils sont tenus pour l'œuvre d'un acteur particulièrement inspiré ou caractériel. Un accent contre-intuitif pourrait être mis également sur des entreprises collectives qui ont échappé à une telle personnalisation. Dans tous les cas, c'est comme truchement et comme révélateur que la catégorie fait sens.

Mobilisée non pas comme révélateur, symptôme ou indice langagier mais pour interpréter un fonctionnement collectif, «école » gagnerait, si l'on suit la suggestion de Jean-Michel Chapoulie dans l'entretien qu'il nous a donné, à être systématiquement complétée, afin de ne pas coller à des usages autochtones. Faute de quoi, le terme incite à reconduire une doxa, qu'il s'agisse de la remotiver ou de la délégitimer, au détriment d'un intérêt pour le vif d'une entreprise. Il a été abondamment question dans ces lignes d' "école de recherche » et d' "école d'activité », comme illustrations (parmi d'autres envisageables) d'un travail spécifique de la «schoolness» — les deux formes générationnelles les plus saillantes en étant le groupe de pairs et le groupe agrégeant apprentis et chevronnés. Il ne s'agit pas ce faisant de favoriser une représentation irénique mettant en exergue les processus coopératifs et de transmission altruiste au détriment de ce qu'il peut y avoir de hiérarchique ou d'intéressé dans de tels dispositifs. En revanche, l'examen du fonctionnement, de la codification, sinon de la ritualisation, voire de l'institutionnalisation, des interactions faciales (ou écrites) sous cadre collectif dans les activités savantes nous semble une perspective stimulante, et dont la mise à jour des traces archivistiques demande de l'ingéniosité (par contraste, elle appellerait plus facilement une ethnographie de pratiques contemporaines).

Suit cette introduction un ensemble de cinq textes prolongés par un entretien, dont il a été donné quelques aperçus. Elles se confrontent à des formules théoriques ou à des interprétations du sens de ce qu'est (ou n'est pas) une "école », certaines penchant nettement du côté de la construction d'un étiquetage savant (textes de M.-C. Robic, J. Carroy et M. Hirschhorn), d'autres s'interrogeant sur une tentative d'institutionnalisation ayant revendiqué cette signalétique particulière, dans un geste rétrospectif ou contemporain (textes de A. Petit et J.-B. Devaux). Elles ne s'arrêtent pas pour autant à cette perspective particulière et tentent de saisir de manière compréhensive des trajectoires ou des moments collectifs. Les «écoles» évoquées sont loin d'avoir toutes été couronnées de succès, en particulier dans la durée, ce qui est une autre tonalité du volume. Parce qu'elle est une expression particulièrement incarnée de collectifs savants — à la différence par exemple de "paradigme » ou de "collège invisible» - la catégorie véhicule souvent une sorte d'implication émotionnelle qui est le propre (supposé) de certaines réalisations collectives, notamment quand elles rencontrent l'échec. C'est sans doute l'une des raisons pour lesquelles elle a tant suscité de passions, de dénégations et de relativisations. 


\section{Bibliographie}

Abbott, A., 1999, Department and Discipline: Chicago Sociology at One Hundred, Chicago, University of Chicago Press.

Abbott, A., 2015, «Actualité de la tradition sociologique de Chicago », Raisons politiques, n 60, p. 9-43.

Albrecht, M., 1995, «Sekte », in Karlfried Gründer (ed.), Historisches Wörterbuch der Philosophie, vol. 9, col. 274-284.

Amsterdamska, O., 1985, "Institutions and Schools of Thought: The Neogrammarians", American Journal of Sociology, Vol. 91, $\mathrm{n}^{\circ} 2$ (September), p. 332-358.

Amsterdamska, O., 1987, Schools of Thought: the Development of Linguistics from Bopp to Saussure, Dordrecht, D. Reidel.

Amsterdamska, O., 1990, «Surely You Are Joking, Monsieur Latour!», Science, Technology and Human Values, XV/4, p. 495-504.

Becker, H., 1999, «The Chicago School, So-Called », Qualitative Sociology, 22 (1), p. 3-12.

Boschetti, A., 2014, Ismes. Du réalisme au postmodernisme, Paris, CNRS Éditions, "Culture \& société ».

Boutier, J., Passeron, J.-C. \& Revel, J., eds, 2006, Qu'est-ce qu'une discipline ?, Paris, éds de l'EHESS, «Enquête ».

Brückle, W., 2003, «II. Stil (kunstwissenschaftlich)», in. Karlheinz Barck, Martin Fontius, Dieter Schlenstedt [et al.], Ästhetische Grundbegriffe, vol. 5 : Postmoderne-Synästhesie, Stuttgart : J. B. Metzler, p. 664-688.

Bulmer, M., 1984, The Chicago School of Sociology, Chicago and London: The University of Chicago Press.

Bulmer, M., 1985, "The Chicago School of Sociology: What Made it a 'School'? ", History of Sociology, 5, p. 61-77.

Carnino, G., 2015, L'Invention de la science. La nouvelle religion de l'âge industriel, Paris, Seuil, «L'univers historique $»$.

Chamboredon, J.-C., 1975, «Sociologie de la sociologie et intérêts sociaux des sociologues », Actes de la recherche en sciences sociales, $\mathrm{n}^{\circ} 2$, p. 2-20.

Chapoulie, J.-M., 2001, La tradition sociologique de Chicago. 1892-1961, Paris, Seuil.

Clark, T. N. \& Clark, P. P., 1971, «Le patron et son cercle : clef de l'Université française », Revue française de sociologie, 12-1. p. 19-39.

Clark, T., 1973, Prophets and patrons, the French University and the Emergence of Social Sciences, Cambridge (Mass.), Harvard University Press.

Collectif, 2015, «Un texte, un auteur : Andrew Abbott», Raisons politiques, n 60, p. 5-64.

Collins, R., 1995, «Les traditions sociologiques », Enquête [En ligne], 2, mis en ligne le 10 juillet 2013, consulté le 25 janvier 2014. URL : http://enquete.revues.org/302 ; DOI : 10.4000 /enquete. 302

Crosland, M., 1967, The Society of Arcueil. A view of French Science at the time of Napoleon I, London, Heinemann.

Demazière, D. \& Jouvenet, M. (dir.), 2016, Andrew Abbott. Sociologue de Chicago, Paris, Presses de l'EHESS

Fabiani, J.-L., 2005, «Faire école en Sciences Sociales. Un point de vue sociologique », Cabiers du CRH, n० 36 p. 191-207.

Fox, R., 1974, "The Rise and Fall of Laplacian Physics ", Historical Studies in the Physical Sciences, Vol. 4, p. 89-136

Geison, G.L., 1978, Michael Foster and the Cambridge School of Physiology: The Scientific Enterprise in Late Victorian Society, Princeton, Princeton UP.

Geison, G.L., 1981, «Scientific Change, Emerging Specialties, and Research Schools », History of Science, XIX-1, p. 20-40. 
Geison, G. L. \& Holmes, eds, 1993, Research Schools: Historical Reappraisals, Osiris, The University of Chicago Press.

Geison, G.L., 1993, "Research Schools and New Directions in the Historiography of Science ", Osiris, vol. 8, p. 226-238.

Gilmore, S., 1988, «Schools of Activity and Innovation », Sociological Quarterly, 29, p. 203-219.

Hannaway, O., 1969, « review of Maurice Crosland, The Society of Arcueil: A View of French Science at the Time of Napoleon I», Isis, 60, p. 578-581.

Harvey, L., 1982, "Use and Abuse of Kuhnian Paradigms in the Sociology of Knowledge », Sociology, 16, p. 85-101.

Harvey, L., 1986, «Myths of the Chicago School», Quality and Quantity, 1986, 20 (2-3), p. 191-217. Harvey, L., 1987a, "The Nature of 'Schools' in the Sociology of Knowledge: The Case of the 'Chicago School' », Sociological Review, p. 245-278.

Harvey, L., 1987b, Myths of the Chicago School of Sociology, Avebury, Aldershot.

Holton, G., 1978, "Fermi's group and the recapture of Italy's place in physics », in The scientific imagination: case studies, p. 155-198.

Hühn, H., Meier-Oeser, S., Pulte, H., 2005, «Wissenschaft», in Gottfried Gabriel (ed.), Historisches Wörterbuch der Philosophie, vol. 12, col. 902-947.

Kaluszynski, M., 2002, La République à l'épreuve du crime: la construction du crime comme objet politique, 1880-1920, LGDJ, « Droit et société ».

Kuhn, T. S., 1959, "The Essential Tension: Tradition and Innovation in Scientific Research », in C. Taylor (ed.), The Third (1959) University of Utah Research Conference on the Identification of Scientific Talent, Salt Lake City, University of Utah Press, p. 162-174.

Kuhn, T. S., 1962/1970, The Structure of Scientific Revolutions, Chicago, University of Chicago Press (1970, 2nd edition, with postscript).

Le Men, S., 2016, «Un art sans frontières ? De 1860 à 1964 » dans Charle, C. \& Jeanpierre, L., La Vie intellectuelle en France 1. Des lendemains de la Révolution à 1914, Paris, Le Seuil, p. 499-524.

Memmi, D., 2008, "Mai 68 ou la crise de la domination rapprochée», in D. Damamme, B. Gobille, F. Matonti, B. Pudal (dir.), Mai-juin 68, Paris, Les Éditions de l'Atelier, p. 35-46.

Merz, J. T., 1965, A History of European Thought in the Nineteenth Century, Vol. I, Scientific Thought (London: Blackwood, 1904), New York, Dover.

Morrell, J. B., 1972, "The Chemist Breeders: The Research Schools of Liebig and Thomson», Ambix, n० 19 , p. 1-46.

Mulkay, M.J. \& Edge, D.O., 1976, Astronomy transformed: the emergence of radio astronomy in Britain, New York, Wiley.

Nye, M.-J., 1993, « National Styles ? French and English Chemistry in the Nineteenth and Early Twentieth Centuries ", Osiris, vol. 8, p. 30-49.

Olesko, K. M., 1993, «Tacit Knowledge and School Formation », Osiris, vol. 8, p. 16-29.

Pudal, R., 2005, « «Sur la réification des collectifs »: à propos de l'école de Chicago », Cahiers internationaux de sociologie, $\mathrm{n}^{\circ} 119$, p. 367-376.

Polanyi, M., 1958, Personal Knowledge: Towards a Post-Critical Philosophy, University of Chicago Press. Polanyi, M., 1966, The Tacit Dimension, London \& Chicago, Routledge \& University of Chicago Press.

Puech, C., 2015, «La notion d'«école linguistique »: unité, singularité, pluralité », Histoire Épistémologie Langage, 37/2, p. 5-15.

Ravetz, J. R., 1979, Scientific knowledge and its social problems, Oxford, Oxford Univ. Press.

Robic, M.-C., 1996, «Interroger le paysage? L'enquête de terrain, sa signification dans la géographie humaine moderne (1900-1950) » dans C. Blanckaert, dir., Le terrain des sciences humaines (XVIII'-XX' siècle), Paris, L'Harmattan, « H.S.H. », p. 357-388.

Robic, M.-C., 1997, «L'excursion du géographe. (Sur l'École française de géographie.), Conférence, $\mathrm{n}^{\circ} 4$, p. 211-227. 
Servos, J. W., 1976, «The Knowledge corporation: A. A. Noyes and chemistry at Cal. Tec., 1915$1930 »$, Ambix, XXIII, p. 175-186.

Servos, J. W., 1993, « Research Schools and Their Histories », Osiris, vol. 8, p. 2-15.

Tiryakian, E., 1979a, "The Significance of Schools in the Development of Sociology ", in W.E. Siznek, E.R. Fuhrmann et M.K. Miller (eds.), Contemporary Issues in Theory and Research. A Metasociological Perspective, Westport, Greenwood, p. 211-233.

Tiryakian, E.,1979b, "The School as The Unit of Analysis: Rethinking the History of Sociology », Ronéotypé.

Tiryakian, EA. "Traditions in Sociology", in N.J. Simelser and P. Baltes, eds, International Encyclopedia of the Social \& Behavioral Sciences, Oxford: Pergamon/Elsevier, 2001, p. 15824-15829.

Topalov, C., 2003, «Écrire l'histoire des sociologues de Chicago », Genèses, n 51, p. 147-159.

Topalov, C., 2004, «Les usages stratégiques de l'histoire des disciplines. Le cas de l'école de Chicago' en sociologie », dans Johan Heilbron, Remi Lenoir et Gisèle Sapiro (ed.), Pour une histoire des sciences sociales. Hommage à Pierre Bourdieu, Paris, Fayard, 2004, p. 127-157.

Ullmann, E. L., 1954, « Geography as spatial interaction », Interregional linkages, the proceedings of the Western Committee on Regional Economic Analysis, Berkeley, California ; reprint in : E. L. Ullman, Geography as spatial interaction (B. R. Boyce editor), Seattle, London, University of Washington Press, 1980.

Waquet, F., 2008, Les enfants de Socrate, Paris, Albin Michel. 
a Né en 1940, Terry Clark a soutenu son PhD à Columbia en 1967. Il fut nommé dès 1966 Assistant Professor à l'université de Chicago, puis Associate Professor en 1972, et enfin Professor en 1985, tout en étant Research Associate au National Opinion Research Center à partir de 1974. Il s'est reconverti assez rapidement dans l'analyse des politiques publiques sous cadre urbain dans leur relation avec la société locale.

b Né en 1929, il a été nommé en 1953 assistant auprès de P. Sorokin. Il soutint son PhD en 1956 sous la direction de T. Parsons. Il a publié en 1962 un ouvrage, Sociologism and existentialism, two perspectives on the individual and society, qui fut un best-seller en Europe de l'Est. Après des positions temporaires à Princeton et Harvard, il fut nommé professeur à Duke University en 1967, où il demeura pour le reste de sa carrière.

c Edward Shils (1910-1995) a été l'une des figures majeures de la sociologie américaine de l'après-guerre, traducteur et «passeur» de Max Weber aux États-Unis, co-signataire de plusieurs ouvrages avec Talcott Parsons, spécialiste du monde intellectuel et universitaire. L'essentiel de son travail a été «métasociologique» (pour paraphraser la terminologie utilisée à son propos) et relève pour une large part de l'essayisme et de la philosophie sociale. Il n'est plus vraiment lu ou même connu en France

d Né en 1943, Martin Bulmer a consacré son travail de thèse à ce qui allait devenir son ouvrage The Chicago School of Sociology sous la direction de Ronald P. Dore et dans un lien étroit avec la London School of Economics. Edward Shils a joué un rôle visiblement important d'interlocuteur intellectuel et de «puits de science » pour son jeune interlocuteur britannique, qui lui rendrait hommage 25 ans plus tard dans son article «Edward Shils as a sociologist » (1996). L'histoire de la sociologie est un de ses champs privilégiés dans une production assez éclectique.

e Lee Harvey est né en 1949. Il est en l'état assez difficile de dresser une esquisse de sa carrière. Sociologue explicitement «critique», longtemps marxiste, il a rendu son œuvre très accessible sur internet (http://www.qualityresearchinternational.com/) mais pas sa biographie. Tout au plus peut-on noter qu'il a été lecturer à University of Central England in Birmingham puis professor à la Sheffield Hallam University (SHU), qu'il a dirigé les revues Quality in Higher Education et Higher Education Quarterly, et s'est occupé à partir de la fin des années 1980 de questions de politique universitaire et de scolarité étudiante. Il a été désavoué par la Higher Education Academy en 2008, suite à une lettre polémique dans le Times Higher Education, épisode qui l'a conduit à démissionner de ses fonctions de directeur du Centre for Research and Evaluation de SHU avant de rejoindre la Copenhagen Business School. Il est notamment l'auteur de Critical Social Research (1990, rééd. 2011), Doing Sociology. A practical introduction (1993, avec Morag MacDonald) et Transforming Higher Education: Students as Key Stakeholders (1996, avec Peter T. Knight).

f Frederic Holmes (1932-2003) a suivi une formation en biologie puis en histoire, avant de soutenir son PhD à Harvard en 1962 sous le titre Claude Bernard and the Concept of the Internal Environment. Il a exercé des fonctions au MIT, à Yale et à Western Colorado University avant de devenir full professor à Yale en 1979. Historien de la médecine et de la chimie, il a exercé de nombreuses responsabilités (notamment éditoriales et administratives) et s'est affirmé comme un organisateur et un pédagogue.

g Gerald Geison (1943-2001) a mené des études de chimie et de philosophie à Beloit College (Wisconsin) avant de faire sa thèse à Yale sous la direction de Frederic Holmes en histoire de la médecine (PhD soutenu en 1970). Il a fait l'ensemble de sa carrière académique à Princeton. Il est surtout connu pour ses travaux sur Louis Pasteur, considérés comme iconoclastes par les défenseurs du culte du grand homme. C'est la préparation d'un autre de ses livres, Michael Foster and the Cambridge School of Physiology: The Scientific Enterprise in Late Victorian Society (1978), qui l'a conduit à investir spécifiquement dans la catégorie de «research school». Il est parfois associé aux rénovateurs des études sur la science parfois désignés comme "école d'Edimbourg» (David O. Edge, Mike Mulkay) ou Social Studies of Knowledge (David Bloor, Barry Barnes, Steven Shapin), mais notre travail a plutôt révélé de la distanciation à leur égard, fût-elle circonstancielle.

h John Servos est né en 1951. Il a fait ses études à Columbia College puis à la John Hopkins University, où il a soutenu son PhD en 1992. Spécialiste au premier chef en histoire de la chimie physique, il est professeur d'histoire à Amherst College (Massachussets), «l'un des établissements les plus libéraux des États-Unis ».

${ }^{i}$ Kathryn M. Olesko est née en 1951, elle a fait toutes ses études à Cornell (licence avec une majeure en physique, master d'histoire), où elle a soutenu son $\mathrm{PhD}$, avant de faire l'essentiel de sa carrière à Georgetown University. Spécialiste de la physique théorique allemande et de ses procédures pédagogiques, elle partage avec John Servos un questionnement sur la place de la science et des technologies dans les sociétés occidentales. Responsable éditoriale d'Osiris, elle a notamment dirigé un numéro sur les femmes dans les sciences.

j Née en 1944, Marie-Jo Nye a commencé ses études à Vanderbilt University, avec une majeure en chimie, puis à Wisconsin University, en master de chimie, avant de faire un $\mathrm{PhD}$ en histoire des sciences dans cette institution sous la direction d'Erwin Hiebert (1970), pour les besoin duquel elle a été témoin du «1968» français, après avoir participé aux «sixties » contestataires américaines. Elle a fait toute sa carrière à University of Oklahoma puis à la State University of Oregon. Spécialiste des développements de la chimie dans la France du XIXe siècle, en particulier provinciale, elle a progressivement évolué vers des perspectives plus épistémologisantes et historiographiques.

k Né en 1933, Jack Morrell a fait l'essentiel de sa carrière comme lecturer (assistant) puis reader (maitre de conférences au sens ancien de professeur sans chaire) à l'université de Bradford (Angleterre). Historien de la chimie anglaise, il a 
diversifié son travail sur les sciences britanniques du côté de l'histoire de la géologie et de l'histoire des institutions savantes. Chercheur assez discret n'ayant jamais disposé d'une position de full professor, il bénéficie par contraste d'une réputation certaine. Il a participé au numéro d'Osiris qui célébrait son article de 1972.

${ }^{1}$ Philosophe des sciences et épistémologue «social», Jerome Ravetz est né en 1929. D’origine américaine mais naturalisé anglais, un temps proche de Stephen Toulmin, il a fait un $\mathrm{PhD}$ de mathématiques avant de devenir le chantre d'une meilleure prise en compte du regard «critique » de la société sur les sciences et de promouvoir une vision "post-normale» (en référence à la catégorie de «science normale chez Kuhn) du développement de ces dernières au temps des risques et de l'incertitude.

m Christian Puech est né en 1950. Il a fait des études de philosophie et de lettres modernes dans les années 1970, avant de s'engager dans une thèse de sciences du langage intitulée La " généralité » entre linguistique et philosophie, sous la direction de Michel Arrivé (soutenue en 1991). Spécialiste de Saussure et de ce qu'il appelle lui-même les saussurismes (son HDR s'intitule Saussurisme et structuralisme : constitution, transmission, diffusion des savoirs linguistiques, 2002), il est l'un des piliers de la Société d'histoire et d'épistémologie des sciences du langage (SHESL) depuis le milieu des années 1990.

${ }^{n}$ Olga Amsterdamska (1953-2009) a fui la Pologne avec sa famille à la fin des années 1960 avant de faire des études en Angleterre (London School of Economics) et aux États-Unis (Yale) : licenciée en sociologie et philosophie, elle a fait son PhD à Columbia sous la direction de Robert Merton, qu'elle a soutenu en 1984 et dont fut tiré son ouvrage de 1987. S'étant installée aux Pays-Bas en 1984 pour un "postdoctoral fellowship» au Department of Science Dynamics de l'université d'Amsterdam, elle y a fait toute sa carrière jusqu'à son décès prématuré, abandonnant l'histoire de la linguistique pour une histoire des sciences centrée sur la biomédecine et la génétique. Elle a participé aux «science wars» par une recension furibonde dans Science, Tecbnology and Human Values (1990) du livre de B. Latour, Science in Action (1987), intitulée « Surely You Are Joking, Monsieur Latour! » (Amstedamska, 1990).

- Né en 1951, normalien de la rue d’Ulm, philosophe de formation, Jean-Louis Fabiani s'est réorienté vers la sociologie, d'abord dans le sillage de P. Bourdieu et de J.-C. Passeron, avant de développer notamment une sociologie historique des « configurations de savoir » interrogeant des catégories génériques comme «discipline » ou «tradition» repensées comme des «institutions savantes».

p Né en 1941, Randall Collins a débuté ses études universitaires à Harvard, au moment où triomphait la «social theory » de Talcott Parsons. À l'issue d'un parcours sinueux, il a entrepris une thèse à Berkeley, soutenue en 1969. Durant ses années à Berkeley, il a particulièrement fréquenté Erving Goffman, Joseph Ben David et Reinhard Bendix (1916-1991), sociologue d'origine allemande, passé par Chicago et l'un des principaux médiateurs de la réception de Max Weber aux États-Unis. R. Collins a également participé aux mouvements protestataires de la fin des années 1960. Une bonne partie de sa production est consacrée à une microsociologie des conflits, de la violence et des interactions face à face entre individus. Une autre part importante de sa production, beaucoup plus théoriciste, relève d'une « métasociologie » et analyse les chaînages d'influence constituant des « traditions ».

q Né en 1948, Andrew Abbott a commencé ses études à Phillips Academy puis Harvard avec une majeure en histoire et littérature, avant de terminer sa formation en sociologie à Chicago, où il a soutenu un $\mathrm{PhD}$ sur la professionnalisation de la psychiatrie sous la direction de Morris Janowitz - l'inventeur de la collection «The Heritage of Sociology», qui a largement participé à la construction rétrospective d'une "école de Chicago ». Historien de la sociologie, méthodologue, spécialiste du « système des professions », parmi d'autres orientations, il bénéficie depuis quelques années d'une réception très favorable en France, cf. Raisons politiques, 2015/4 et Demazière et Jouvenet, dir., 2016.

${ }^{\mathrm{r}}$ Né en 1928, Howard Becker a fait ses études à l'université de Chicago dans les années d'après-guerre, revendiquant lui-même des influences multiples dans ce cadre (Everett Hughes, William Lloyd Warner, beaucoup moins Herbert Blumer ou Louis Wirth). Il a été professeur à la Northwestern puis à University of Washington (Seattle). Ses travaux de sociologie de la déviance (Outsiders, 1973) insistent sur les processus de négociation des cadres interprétatifs amenant des «entrepreneurs de morale» à tenter d'imposer une certaine lecture qui étiquette et stigmatise des déviants. Ces derniers sont soustraits par lui à un quelconque déterminisme par une attention à leur trajectoire, réinterprétée en termes de "carrière» et d'apprentissage pratique. Rétif aux étiquetages (notamment celui d' «interactionnisme symbolique » qui procède d'H. Blumer), il a produit une œuvre polymorphe s'intéressant notamment aux « mondes de l'art » et à l'écriture de la sociologie. cf. http://howardsbecker.com/ 